\title{
Morning Peak-Period Pricing Surcharge of Elderly Passengers Taking Express Buses
}

\author{
Jingxu Chen, ${ }^{1,2,3}$ Chengxin He, ${ }^{1}$ Xinlian Yu $\mathbb{D}^{1,2}$ and Wendong Chen ${ }^{1}$ \\ ${ }^{1}$ Jiangsu Key Laboratory of Urban ITS, Jiangsu Province Collaborative Innovation Center of Modern Urban Traffic Technologies, \\ Southeast University, Si Pai Lou No. 2, Nanjing, China \\ ${ }^{2}$ School of Transportation, Southeast University, Nanjing, China \\ ${ }^{3}$ Department of Logistics and Maritime Studies, The Hong Kong Polytechnic University, Hong Kong, China
}

Correspondence should be addressed to Xinlian Yu; xinlianyu@seu.edu.cn

Received 12 January 2021; Revised 5 August 2021; Accepted 2 September 2021; Published 29 September 2021

Academic Editor: Luigi Dell'Olio

Copyright (c) 2021 Jingxu Chen et al. This is an open access article distributed under the Creative Commons Attribution License, which permits unrestricted use, distribution, and reproduction in any medium, provided the original work is properly cited.

\begin{abstract}
This study deals with the elderly fare pricing issue for taking express buses in the morning peak period. As many elderly passengers are not commuters, fare discount policy may not be an opportune option when buses get overcrowded. Imposing surcharge on the elderly becomes a potentially beneficial measure that encourages an appropriate number of elderly passengers to circumvent the most crowded buses. The elderly pricing surcharge problem is formulated as a bilevel model, in which the upper-level model is to make the pricing surcharge decision, and the lower-level model is the equilibrium passenger assignment that represents passengers' bus choice behavior. It is classified into the special case and the generic case depending on the number of buses that impose surcharge. Several useful properties of two cases are analyzed, and a trial-and-error solution method is later developed to solve these two cases. Numerical experiments show that the elderly pricing surcharge scheme is not always applicable to all the demand scenarios, which owns a certain effective interval.
\end{abstract}

\section{Introduction}

Public transit exerts a crucial part in sustainable urban development, which is recognized as an effective way to alleviate the problems attributed to the automobile, such as road congestion and air pollution. In the past decades, transit planners and operators are devoted to enhancing service efficiency and attractiveness by introducing a wide variety of instruments that encompass governmental policies, planning process, and control strategies (e.g., [1-10], among many others). For bus service, fare discount policy is commonly implemented in cities worldwide, e.g., the elderly are privileged to take buses using discount bus tickets. This policy instrument aims to attract more elderly passengers and hence increase ridership. Since aging society is emerging as a ubiquitous phenomenon, fare subsidy is conducive to cultivating the elderly passenger demand in the long run.

The analysis pertinent to spatiotemporal travel characteristics of the elderly is now receiving increasing attention
$[11,12]$. The elderly people's travel modes vary between different countries and regions; for example, the most frequently used mode by the elderly is car in western developed countries whereas it becomes public transit in Asian cities (e.g., Hong Kong and Seoul). In the public transit-oriented city, elderly passengers generally prefer to use the transit service during off-peak hours so as to stay away from commuting crowds in peak period [13]. Nevertheless, Shao et al. [14] presents an intriguing finding that contradicts this generic rule. They find that the percentage of elderly people taking buses is similar with that of younger people (around $10 \%)$ during the morning peak period. It is probably that many elderly passengers want to buy cheaper and fresher foodstuffs at morning markets in other communities or to exercise at a faraway park.

Express bus is a bus service that may manifest the above phenomenon; that is, both adults with full-price ticket and elderly passengers have analogous percentage to choose. It is primarily intended to operate between residential 
communities and city centers (e.g., central business district or CBD for short). Many passengers using express buses usually travel on a daily commuting basis, and meanwhile, some elderly passengers arrive at morning markets or parks in city centers. When express buses have large passenger demand during the morning peak period, a part of passengers alter their departure time choice in order to board less crowded neighboring buses. Nonetheless, the level of service for certain buses is still low which engenders passenger complaint due to the overcrowded riding experience. Current fare discount policy may be no more appropriate for this pattern of bus service since trip purposes of elderly passengers are varied, and many of them belong to the noncommuting travel (e.g., recreational activities). In contrast, imposing surcharge on the elderly is a potential measure that encourages some elderly passengers to circumvent the most crowded buses. This paper focuses on the elderly pricing surcharge problem.

1.1. Literature Review. Policy instruments for public transit service aim at increasing the transit serviceability and attractiveness. Fare-related policy is one of the most common and effective policy instruments for transit operation management [15].

A large number of studies have been conducted on the issue of transit fare change from different points of view. For example, Nuworsoo et al. [16] compared the effect of different fare increase options on social equity based upon onboard survey data from the agency AC Transit, Canada. Nahmias-Biran et al. [17] used the Gini index to examine whether fare changes were equally distributed among different passenger groups. From the angle of accessibility, Ma et al. [18] applied the cumulative opportunity measure and found that the loss in work accessibility resulting from transit fare increase is inversely proportional to trip length, given the flat fare structure. In the work of Yang and Tang [19], the difference-in-differences model was utilized to assess the influence of transit fare increase on environment using data of Beijing, China. Results show that fare increase yields around $16 \%$ growth of air pollution in the short term, while it has no long-run effect on air quality. In addition, fare change is carried out in association with other transit policies, and several studies have investigated the impact of the broader policy change on ridership, revenue, and passengers' travel behavior (e.g., [20-23]).

Various time-based pricing schemes which apply to a specific time of day have been studied by several scholars as well. For example, Lovrić et al. [24] proposed the activitybased demand model to evaluate two off-peak discount schemes and found that two schemes assist in spreading demand peaks, especially the afternoon peak period. In the work of Huang et al. [25], the in-vehicle crowding effect and passenger's departure time choice were considered. They developed the bottleneck model to analyze the passenger flow distribution at equilibrium of transit service with two stations and discussed the impact of fare policies. Tian et al. [26] extended this work to explore the equilibrium properties of passenger flow for the many-to-one transit system.
Yang and Tang [27] proposed a new fare-reward scheme for managing passengers' departure time choice in a rail transit bottleneck, which aims to shift partial passenger demand from peak period to off-peak period.

With an aging baby boomer population, elderly mobility is becoming an increasingly important social issue, which has aroused great interest in the research community. In recent years, researchers have studied the issue of elderly mobility from various perspectives, including public transit accessibility [28, 29], pedestrian environment [30, 31], driving behaviors $[32,33]$, and crossing road intersections $[34,35]$, among many others. Some of these studies have also reported many beneficial findings on the differences between elderly users in the different parts of the world. Furthermore, some studies have been dedicated to the transit service of elderly passengers. Newmark [36] compared 10 representative cities in the US that implement fare discount policies for the elderly and qualitatively discussed the attendant tradeoffs. Wong et al. [37] evaluated the level of satisfaction with different public transport modes using interview survey data conducted among elderly passengers in Hong Kong, China. Based on bus passenger survey in Harbin, China, Yuan et al. [38] employed the structural equation model to evaluate the importance of ten dimensions of bus service quality for elderly passengers. These findings are useful for understanding the elderly travel behaviors. However, none of the previous studies have explored the time-based pricing schemes pertinent to elderly passengers particularly. Current fare discount policy may not be a suited option for the crowded bus service with a large quantity of commuters. (Some studies show the need to protect the elderly using public transit. The elderly is regarded as a less privileged population with limited transport choices, and their mobility is dependent on public transit in the public transit-oriented city [29]. Fare discount policy is a way to protect the elderly, which should never be readily canceled. Meanwhile, the elderly have significantly less commuting and work-related trips as compared to younger people. During the morning peak period, imposing surcharge on some particular buses is a worthwhile attempt that encourages elderly passengers to actively circumvent the most crowded buses (these buses are mainly used for commuting). In such case, elderly passengers still enjoy fare discounts when taking the remaining buses.) Crowding worsens the quality of bus service and its perception [7, 39], Björklund and Swärdh, [40], and it also harms the health of passengers, especially the elderly. Therefore, an analysis of the peak-hour fare pricing for the elderly would be beneficial to potentially ameliorate the invehicle crowding effect during the morning peak period.

1.2. Objectives and Contributions. The main objective and contribution of this study are to propose a methodology for determining the elderly fare pricing for taking express buses in the morning peak period. It aims to acquire the optimal pricing surcharge of elderly passengers in comparison with the current fare discount policy. A bilevel optimization model is developed in which the upper-level model is to make the pricing surcharge decision, and the lower-level 
model is the equilibrium passenger assignment that represents passengers' bus choice behavior. The elderly pricing surcharge (EPS) problem is classified into the special case and the generic case depending on the number of buses that need to pay additional fare by the elderly passengers. Later, we develop a trial-and-error solution method to solve two EPS cases. It is capable of considering some practical circumstances (e.g., the crowding effect) without requiring their explicit form.

The remainder of this paper is organized as follows: Section 2 describes the problem and the bilevel optimization model. The solution method is introduced in Section 3. Section 4 depicts illustrative examples. Finally, conclusions are provided in Section 5.

\section{Problem Description and Model Development}

Consider an express bus service between a particular residential community and the CBD. Passengers are divided into two categories. We indicate by $k=1$ the first category (adults with full-price ticket) and by $k=2$ the second category (the elderly with discount-price ticket). Variables used in the model and their notation are summarized in Table 1 (see Appendix B). The associated ticket prices of these two passenger categories are denoted by $p_{1}$ and $p_{2}$, respectively. Suppose that the timetable of bus service is predefined, and buses are dispatched at a constant headway (denoted by $h$ ). Let $\Lambda=\{-\xi, \ldots,-2,-1,0,1,2, \ldots, \zeta\}$ be the set of dispatched buses, and $i \in \Lambda$ denote each bus vehicle. Parameters $\xi$ and $\zeta$ in set $\Lambda$ are sufficiently large to cover the entire morning peak period.

Each passenger has his/her own desired time arriving at the CBD which is coherent with the destination arrival time of one bus vehicle in set $\Lambda$. Let $q_{i}^{1}$ and $q_{i}^{2}$ represent the number of adult passengers with full-price ticket and elderly passengers whose desired bus is $i \in \Lambda$, respectively. Let $q_{i}=q_{i}^{1}+q_{i}^{2}$. Suppose that $q_{i}^{k}$ in set $\Lambda(k \in\{1,2\})$ exhibits a weakly unimodal distribution, i.e., $q_{i}^{k} \leq q_{i+1}^{k}$ when $-\xi \leq i \leq-$ 1 and $q_{i}^{k} \leq q_{i-1}^{k}$ when $1 \leq i \leq \zeta$. It signifies that $q_{0}=\max _{i \in \Lambda}\left\{q_{i}\right\}$. Hence, bus 0 and its neighboring buses are more likely to be in-vehicle crowded. Let $-\bar{\xi}(\bar{\xi}<\xi)$ and $\bar{\zeta}$ $(\bar{\zeta}<\zeta)$ represent the first bus and the last bus whose $q_{i}$ already make the vehicle become crowded, respectively (e.g., exceed the number of seats). Define $\bar{\Lambda}=\{-\bar{\xi}, \ldots,-2,-1,0,1,2, \ldots, \bar{\zeta}\}$ and use subscript $j$ to denote crowded bus in set $\bar{\Lambda}$. Note that set $\bar{\Lambda}$ is known provided that the values of $q_{i}$ in set $\Lambda$ are given. Pricing surcharge scheme of elderly passengers is assumed to be implemented on the buses in set $\bar{\Lambda}$.

For analytical tractability, we assume that all the passengers have the identical value of travel time and valuation of in-vehicle crowding. Let $\tau$ denote the travel time between the suburban area and the $\mathrm{CBD}$, which is assumed to be constant for each bus. The unit cost of passenger in-vehicle travel time is denoted by $\alpha$. For passengers whose desired bus is $j \in \bar{\Lambda}$, they may take the neighboring buses instead of bus $j$ since bus $j$ is crowded. Then, $i<j$ means that passengers select bus $i$ which arrives early than its desired bus $j$ and the early arrival time is $-h(i-j)$, while $i>j$ means that the selected bus $i$ arrives late and the late arrival time is $h(i-j)$. Let $\beta$ and $\gamma$ denote the schedule delay penalty of unit early and late arrival time, respectively. When passengers whose desired bus is $j \in \bar{\Lambda}$ select bus $i \in \Lambda$, the early/late arrival penalty cost $\delta_{i, j}$ can be expressed as

$$
\delta_{i, j}= \begin{cases}-\beta h(i-j), & i<j, \\ 0, & i=j, \\ \gamma h(i-j), & i>j .\end{cases}
$$

We define $n_{i, j}^{1}$ and $n_{i, j}^{2}$ as the number of adults with fullprice ticket and elderly passengers taking bus $i \in \Lambda$ while their desired bus is $j \in \bar{\Lambda}$, respectively. In this study, the crowding cost is further taken into account, which is associated with the crowdedness (denoted by $g$ ) and the invehicle travel time $\tau$. Here, $g$ is a monotonically increasing function of in-vehicle passenger flow and $g(0)=0$. We define $N_{i}$ as the number of passengers selecting bus $i$. Then, the crowding cost of taking bus $i$ per passenger (denoted by $C_{i}$ ) is given by

$$
C_{i}=\tau g\left(N_{i}\right)
$$

The elderly pricing surcharge (EPS) problem tries to shift an appropriate number of elderly passengers to less crowded buses by using fare instrument. It can be represented as a leader-follower game where the local authority makes the elderly pricing surcharge decision, which can affect, but cannot control the passengers' bus choice behavior. The passengers in two categories make choices with regard to their selection of buses in order to minimize their individual travel cost. Hence, EPS can be formulated as a bilevel model as shown below.

\subsection{Upper-Level Model [UM].}

$\min \rho$,

subject to

$$
\begin{gathered}
N_{i} \leq \phi Q, \quad i \in \Lambda, \\
0 \leq \rho \leq p_{1}-p_{2} .
\end{gathered}
$$

In model [UM], objective function (3) is to determine the minimum amount of fare increase for elderly passengers who board crowded buses in set $\bar{\Lambda}$ (denoted by the decision variable $\rho$ ). Note that the ticket price remains unchanged (i.e., $p_{2}$ ) if the elderly choose buses in set $\Lambda / \bar{\Lambda}$. Equation (4) ensures that the number of passengers in bus $i$ should be maintained to a certain level of service, where $\phi$ is a constant parameter indicating the threshold of crowdedness degree and $Q$ denotes the bus capacity (i.e., the number of seats plus standing capacity). Equation (5) restricts the range of fare increase, namely, the elderly ticket price $p_{2}+\rho$ should not exceed the full ticket price $p_{1}$.

Define $\quad \mathbf{n}=\left\{n_{i, j}^{k} \mid i \in \Lambda, j \in \bar{\Lambda}, k=1,2\right\} \quad$ and $\mathbf{N}=\left\{N_{i} \mid i \in \Lambda\right\}$. The lower-level model is the passenger assignment problem which represents passengers' bus choice 
TABLE 1: List of notation.

\begin{tabular}{|c|c|}
\hline Variable & Description \\
\hline$i, j$ & The index of dispatched buses \\
\hline$k$ & $\begin{array}{c}\text { The index of two passenger categories ( } k=1 \text { denotes adults with full-price ticket, and } k=2 \text { denotes the elderly with discount- } \\
\text { price ticket) }\end{array}$ \\
\hline$\Lambda$ & $\begin{array}{c}\text { A set of dispatched buses } \Lambda=\{-\xi, \ldots,-2,-1,0,1,2, \ldots, \zeta\} \text { where }-\xi \text { and } \zeta \text { denote the first bus and the last bus in the morning } \\
\text { peak period, respectively }\end{array}$ \\
\hline $\bar{\Lambda}$ & $\begin{array}{r}\text { A set of dispatched buses } \bar{\Lambda}=\{-\bar{\xi}, \ldots,-2,-1,0,1,2, \ldots, \bar{\zeta}\} \text { where }-\bar{\xi} \text { and } \bar{\zeta} \text { denote the first bus and the last bus whose } q_{i} \text { already } \\
\text { make the vehicle become crowded (e.g., exceed the number of seats), respectively }\end{array}$ \\
\hline$p_{1}$ & The ticket price of the first category (adults with full-price ticket) \\
\hline$p_{2}$ & The ticket price of the second category (the elderly with discount-price ticket) \\
\hline$q_{i}^{1}$ & The number of adult passengers with full-price ticket whose desired bus is $i \in \Lambda$ \\
\hline$q_{i}^{2}$ & The number of elderly passengers whose desired bus is $i \in \Lambda$ \\
\hline$q_{i}$ & The number of passengers whose desired bus is $i \in \Lambda, q_{i}=q_{i}^{1}+q_{i}^{2}$ \\
\hline$h$ & Constant headway of dispatched buses \\
\hline$\tau$ & The travel time between the suburban area and the CBD \\
\hline$\alpha$ & The unit cost of passenger in-vehicle travel time \\
\hline$\phi$ & The threshold of bus crowdedness degree \\
\hline$\delta_{i, j}$ & The arrival penalty cost when passengers whose desired bus is $j \in \bar{\Lambda}$ select bus $i \in \Lambda$ \\
\hline$\beta$ & The schedule delay penalty of unit early arrival time \\
\hline$\gamma$ & The schedule delay penalty of unit late arrival time \\
\hline$n_{i, j}^{1}$ & The number of full-price adults taking bus $i \in \Lambda$ while their desired bus is $j \in \bar{\Lambda}$ \\
\hline$n_{i, j}^{2}$ & The number of elderly passengers taking bus $i \in \Lambda$ while their desired bus is $j \in \bar{\Lambda}$ \\
\hline$g$ & A monotonically increasing function of in-vehicle passenger flow and $g(0)=0$ \\
\hline$\rho$ & The pricing surcharge of elderly passengers who board crowded buses in set $\bar{\Lambda}$ \\
\hline
\end{tabular}

behavior. Its decision is to garner two optimal vectors $\mathbf{n}$ and $\mathbf{N}$ at equilibrium (denoted by $\mathbf{n}^{*}$ and $\mathbf{N}^{*}$, respectively). Here, we make one assumption in regard to passengers whose desired bus $i$ is in set $\Lambda / \bar{\Lambda}$ : they are always available to board their desired bus regardless if other passengers from set $\bar{\Lambda}$ select to take bus $i$ or not. (Due to crowding effect, passengers whose desired bus $i \in \bar{\Lambda}$ may shift to the neighboring uncrowded bus like bus $-\bar{\xi}-1$. Once bus $-\bar{\xi}-1$ gets crowded, some of them may take $-\bar{\xi}-2,-\bar{\xi}-3$... The assumption indicates that passengers whose desired bus $i \in \Lambda / \bar{\Lambda}$ (e.g., bus $-\bar{\xi}-1$ ) owns the priority to firstly board bus $i$, and hence, they are always board their desired bus. In other words, only passengers whose desired bus $i \in \bar{\Lambda}$ may shift to bus $-\bar{\xi}-1$ and even earlier buses. It is amenable to the case of late dispatched bus $\bar{\zeta}+1$ as well. In reality, the first boarding priority does not exist, and some passengers whose desired bus $i \in \Lambda / \bar{\Lambda}$ may also possibly shift to less crowded buses due to the influx of sufficient passengers from set $\bar{\Lambda}$. Nonetheless, this somewhat strong assumption can be relaxed which will be particularly discussed in Proposition 3 of Section 2.1.) Under this assumption, the ticket fare and invehicle travel cost of passengers whose desired buses correspond to set $\Lambda / \bar{\Lambda}$ is a constant value (i.e., $\left.\sum_{k=1}^{2} \sum_{i \in \Lambda / \Lambda} q_{i}^{k}\left(p_{k}+\alpha \tau\right)\right)$ which is hence not considered. It is noteworthy that the crowding cost resulted from these passengers is still influenced by $\rho$, which should be taken into account.

\subsection{Lower-Level Model [LM]}

$$
\left(\mathbf{n}^{*}, \mathbf{N}^{*}\right):=\arg \min _{\mathbf{n}, \mathbf{N}} \sum_{i \in \Lambda}\left(\sum_{k=1}^{2} \sum_{j \in \bar{\Lambda}} n_{i, j}^{k}\left(p_{k}+\alpha \tau+\delta_{i, j}\right)+\sum_{j \in \bar{\Lambda}} n_{i, j}^{2} \rho+\tau G\left(N_{i}\right)\right)
$$

subject to

$$
\begin{aligned}
& \sum_{i \in \Lambda} n_{i, j}^{k}=q_{j}^{k}, \quad j \in \bar{\Lambda}, k=1,2, \\
& N_{i}= \begin{cases}\sum_{k=1}^{2} \sum_{j \in \bar{\Lambda}} n_{i, j}^{k}, & i \in \bar{\Lambda}, \\
\sum_{k=1}^{2} \sum_{j \in \bar{\Lambda}} n_{i, j}^{k}+q_{i}, & i \in \Lambda / \bar{\Lambda},\end{cases}
\end{aligned}
$$

$$
\begin{array}{r}
n_{i, j}^{k} \geq 0, \quad i \in \Lambda ; j \in \bar{\Lambda} ; k=1,2, \\
N_{i} \geq 0, \quad i \in \Lambda .
\end{array}
$$

In model [LM], objective function (6) contains five cost terms. The first three terms are ticket fare, in-vehicle travel cost, and schedule delay penalty cost of passengers whose desired buses are in set $\bar{\Lambda}$, respectively. The fourth term indicates the surcharge for the elderly taking buses in set $\bar{\Lambda}$. The last term is a derivative of Beckmann's transformation [41], which signifies the sum of the integrals of the in-vehicle 
crowding costs where $G(x)$ equals $\int_{0}^{x} g(\omega) \mathrm{d} \omega$. Similar to the user equilibrium models $[41,42]$, the term $G(x)$ does not have any economic interpretation. Equation (7) exhibits that the sum of passengers in category $k$ whose desired bus $j \in \bar{\Lambda}$ equal $q_{j}^{k}$. Equation (8) couples the variables $n_{i, j}^{k}$ and $N_{i}$. Equations (9) and (10) enforce nonnegativity of variables.

Replacing $N_{i}$ in equations (6) by (8), the lower-level model only contains decision variables $n_{i, j}^{k}$

$$
\mathbf{n}^{*}:=\arg \min _{\mathbf{n}}\left(\begin{array}{l}
\sum_{i \in \Lambda}\left(\sum_{k=1}^{2} \sum_{j \in \bar{\Lambda}} n_{i, j}^{k}\left(p_{k}+\alpha \tau+\delta_{i, j}\right)+\sum_{j \in \bar{\Lambda}} n_{i, j}^{2} \rho\right) \\
+\sum_{i \in \bar{\Lambda}} \tau G\left(\sum_{k=1}^{2} \sum_{j \in \bar{\Lambda}} n_{i, j}^{k}\right)+\sum_{i \in \Lambda \backslash \bar{\Lambda}} \tau G\left(\sum_{k=1}^{2} \sum_{j \in \bar{\Lambda}} n_{i, j}^{k}+q_{i}\right)
\end{array}\right),
$$

subject to

$$
\begin{aligned}
& \sum_{i \in \Lambda} n_{i, j}^{k}=q_{j}^{k}, \quad j \in \bar{\Lambda}, k=1,2, \\
& n_{i, j}^{k} \geq 0, \quad i \in \Lambda ; j \in \bar{\Lambda} ; k=1,2 .
\end{aligned}
$$

$$
\begin{gathered}
n_{i, j}^{1}\left(p_{1}+\alpha \tau+\delta_{i, j}+C_{i}-\lambda_{j}^{1}\right)=0, \quad i \in \Lambda ; j \in \bar{\Lambda}, \\
p_{1}+\alpha \tau+\delta_{i, j}+C_{i}-\lambda_{j}^{1} \geq 0, \quad i \in \Lambda ; j \in \bar{\Lambda}, \\
n_{i, j}^{2}\left(p_{2}+\rho+\alpha \tau+\delta_{i, j}+C_{i}-\lambda_{j}^{2}\right)=0, \quad i \in \bar{\Lambda} ; j \in \bar{\Lambda}, \\
p_{2}+\rho+\alpha \tau+\delta_{i, j}+C_{i}-\lambda_{j}^{2} \geq 0, \quad i \in \bar{\Lambda} ; j \in \bar{\Lambda}, \\
n_{i, j}^{2}\left(p_{2}+\alpha \tau+\delta_{i, j}+C_{i}-\lambda_{j}^{2}\right)=0, \quad i \in \Lambda \backslash \bar{\Lambda} ; j \in \bar{\Lambda}, \\
p_{2}+\alpha \tau+\delta_{i, j}+C_{i}-\lambda_{j}^{2} \geq 0, \quad i \in \Lambda \backslash \bar{\Lambda} ; j \in \bar{\Lambda}, \\
C_{i}=\left\{\begin{array}{r}
\tau g\left(\sum_{k=1}^{2} \sum_{j \in \bar{\Lambda}} n_{i, j}^{k}\right), \\
\tau g\left(\sum_{k=1}^{2} \sum_{j \in \bar{\Lambda}} n_{i, j}^{k}+q_{i}\right), \quad i \in \Lambda \backslash \bar{\Lambda}
\end{array},\right. \\
\sum_{i \in \Lambda} n_{i, j}^{k}=q_{j}^{k}, \quad j \in \bar{\Lambda}, k=1,2, \\
n_{i, j}^{k} \geq 0, \quad i \in \Lambda ; j \in \bar{\Lambda} ; k=1,2,
\end{gathered}
$$

where $\lambda_{j}^{1}$ and $\lambda_{j}^{2}$ are the Lagrangean multipliers of constraint (12). Equations (14) and (15) unveil that if $n_{i, j}^{1}>0$, the generalized travel cost of taking bus $i$ equals $\lambda_{j}^{1}$ for adults with fullprice ticket whose desired bus is $j \in \bar{\Lambda}$. Otherwise, $n_{i, j}^{1}$ equals zero. Hence, $\lambda_{j}^{1}$ represents the equilibrium generalized travel cost of passengers in the first category. For passengers in the second category, the equilibrium generalized travel cost $\lambda_{j}^{2}$ can be analogously defined. Equations (16) and (17) exhibit the case when elderly passengers whose desired bus is $j \in \bar{\Lambda}$ select one bus in set $\bar{\Lambda}$ while equations (18) and (19) exhibit the case when these passengers select the bus from set $\Lambda / \bar{\Lambda}$. Equation (20) presents the relation between the crowding $\operatorname{cost} C_{i}$ and decision variable $n_{i, j}^{k}$ by substituting equation (8) into (2).

Remark 1. Given the value of $\rho$, the equilibrium generalized travel costs of two passenger categories (i.e., $\left.\lambda_{j}^{k}, j \in \bar{\Lambda}, k \in\{1,2\}\right)$ are uniquely determined. Nonetheless, the optimal solution of the lower-level model is not unique in 
terms of $n_{i, j}^{k}$. In other words, $\lambda_{j}^{k}$ can be achieved through different combinations of $n_{i, j}^{k}$. For example, suppose that in one optimal equilibrium solution, $n_{i=-\bar{\xi}-1, j=-\bar{\xi}}^{k}>0$ and $n_{i=-\bar{\xi}-2, j=-\bar{\xi}}^{k}>0$ for $k=1,2$. Let $n_{i}^{\min }=\min \left\{n_{i, j=-\bar{\xi}}^{k} \mid i=-\bar{\xi}-1\right.$, $-\bar{\xi}-2 ; k=1,2\}$. For passengers whose desired bus is $-\bar{\xi}, n_{i}^{\min }$ elderly passengers taking bus $-\bar{\xi}-1$ and $n_{i}^{\min }$ passengers with full-price ticket selecting bus $-\bar{\xi}-2$ exchange positions with each other. It is easy to verify the new solution of $n_{i, j}^{k}$ yielded by the above operation is one optimal solution as well.

2.3. A Special Case with One Bus in Set $\bar{\Lambda}$. We first discuss a special case that set $\bar{\Lambda}$ only contains one bus vehicle 0 (i.e., $\bar{\Lambda}=\{0\})$. As per the definition of $\bar{\Lambda}$, passengers whose desired bus is bus 0 are most likely to select the neighboring bus vehicles.

Proposition 1. When $\rho>0, n_{i=0, j=0}^{1}>0$ always holds in the optimal equilibrium solution.

Proposition 2. If passengers whose desired bus $j=0$ select bus $i \leq-2$ (i.e., $n_{i, j=0}^{1}+n_{i, j=0}^{2}>0$ ), the equilibrium generalized travel costs of taking bus $i^{\prime}=i, \ldots-2,-1$ are identical for each passenger category $k(k \in\{1,2\})$.

The proofs of Proposition 1 and 2 are provided in Appendixes $\mathrm{A}$ and $\mathrm{B}$, respectively.

Lemma 1. If $n_{i, j=0}^{1}+n_{i, j=0}^{2}>0 \quad(i \geq 2)$, passengers in each category $k(k \in\{1,2\})$ whose desired bus $j=0$ share the same generalized travel cost when taking bus $i^{\prime}=1,2, \ldots, i$.

Lemma 2. If $\sum_{i \in \Lambda / \Lambda} n_{i, j=0}^{k}>0, k \in\{1,2\}$, at least one of bus -1 and bus 1 contains passengers in category $k$ whose desired bus $j=0$ at equilibrium.

Remark 2. If $n_{i, j=0}^{1}>0$ where $i \in \Lambda / \bar{\Lambda}$, the equilibrium generalized travel costs of taking bus $i$ and bus 0 are the same for passengers with full-price ticket whose desired bus $j=0$. In contrast, if $n_{i, j=0}^{2}>0$ where $i \in \Lambda / \bar{\Lambda}$ at equilibrium, the generalized travel cost of taking bus $i$ is less than (or equal to) that of taking bus 0 for elderly passengers whose desired bus $j=0$ when $\rho>0$.

Proposition 3. For passengers whose desired bus is in set $\Lambda \backslash \bar{\Lambda}$, the assumption that they are always available to board their desired bus can be relaxed, with no influence on the objective value of the lower-level model.

The proof of Proposition 3 is provided in Appendix C. Here, the original condition $\rho=0$ is analyzed. When $\rho$ equals 0 , we indicate by $\bar{n}_{i, j=0}^{k}$ the optimal value of $n_{i, j=0}^{k}$ and by $\bar{\lambda}_{j=0}^{k}$ the generalized travel cost of passengers in category $k$ whose desired bus is bus 0 . In this study, we assume that there exist both passengers with full-price ticket and elderly passengers in bus 0 at equilibrium when $\rho=0$, namely, $\bar{n}_{i=0, j=0}^{1}>0$ and $\bar{n}_{i=0, j=0}^{2}>0$. Therefore, the equilibrium generalized travel cost $\lambda_{j=0}=p_{1}+\alpha \tau+C_{0}$ and hold. The relation between $\bar{\lambda}_{j=0}^{2}=p_{2}+\alpha \tau+C_{0}$ and $\bar{\lambda}_{j=0}^{1}$ is $\bar{\lambda}_{j=0}^{2}$.
Based upon the value of $\bar{\lambda}_{j=0}^{1}-p_{1}=\bar{\lambda}_{j=0}^{2}-p_{2}$, the special case with single bus in set $\bar{n}_{i=0, j=0}^{k}$ can be further decomposed into two scenarios when $\bar{\Lambda}$. Proposition 4 gives the $\rho>0$ equilibrium solution of the lower-level model under two scenarios.

Proposition 4. Scenario $I\left(q_{0}^{1}-\bar{n}_{i=0, j=0}^{1}>\bar{n}_{i=0, j=0}^{2}\right), \lambda_{j=0}^{1}-p_{1}=$ $\lambda_{j=0}^{2}-p_{2}$ and $n_{i=0, j=0}^{2}=0$ hold at equilibrium given that $\rho>0$; Scenario II $\left(q_{0}^{1}-\bar{n}_{i=0, j=0}^{1} \leq \bar{n}_{i=0, j=0}^{2}\right), \lambda_{j=0}^{1}-p_{1} \geq \lambda_{j=0}^{2}-p_{2}-\rho$ and $\sum_{i \in \Lambda / \Lambda} n_{i, j=0}^{1}=0$ hold at equilibrium given that $\rho>0$.

The proof of Proposition 4 is provided in Appendix D.

Remark 3. As indicated in Remark 1, the equilibrium solution in the case of $\rho=0$ is unique with respect to $\bar{\lambda}_{j}^{k}$, but not unique with respect to $\bar{n}_{i, j}^{k}$. Due to the monotonicity of $C_{i}$ in equation (2), $\bar{N}_{i}$ is uniquely determined as well. Therefore, two scenarios in Proposition 4 can be rephrased as follows: Scenario I $\left(q_{0}^{1}>\bar{N}_{0}\right)$ and Scenario II $\left(q_{0}^{1} \leq \bar{N}_{0}\right)$.

Remark 4. In Scenario I, as $n_{i=0, j=0}^{2}$ always equals zero, $\rho$ is irrelevant to the generalized travel cost of two passenger categories provided that $\rho>0$; in Scenario II, $n_{i=0, j=0}^{1}$ equals $q_{0}^{1}$, and we can easily verify that an increment of $\rho$ will result in a monotonic decrease in the value of $n_{i=0, j=0}^{2}$ until $n_{i=0, j=0}^{2}$ equals zero.

\subsection{A Generic Case with Multiple Buses in Set $\bar{\Lambda}$}

2.4.1. $\rho$ Equals 0. Regarding the generic case with multiple buses in set $\bar{\Lambda}$, we firstly analyze the original condition $\rho=0$. Consider a hypothetical circumstance that bus capacity is sufficiently large; for example, the number of seats is larger than $\max _{i \in \Lambda}\left\{q_{i}\right\}$. In such ideal circumstance, all the passengers will unhesitatingly take their desired buses with no concern of in-vehicle crowding. At equilibrium, the number of passengers selecting bus $i$ (i.e., $N_{i}$ ) presents the same distribution as $q_{i}$ for buses in set $\Lambda$.

In the hypothetical circumstance, we then gradually reduce bus capacity. Buses that carry $\max _{i \in \Lambda}\left\{q_{i}\right\}$ passengers are the first to be crowded. (In this study, $q_{i}^{k}$ in set $\Lambda(k \in\{1,2\})$ is assumed to follow the weakly unimodal distribution, i.e., $q_{i}^{k} \leq q_{i+1}^{k}$ when $-\xi \leq i \leq-1$ and $q_{i}^{k} \leq q_{i-1}^{k}$ when $1 \leq i \leq \zeta$. It is possible that apart from bus $0, q_{i}$ of some neighboring buses (e.g., bus -1 or bus 1 ) may also equal $\max _{i \in \Lambda}\left\{q_{i}\right\}$.) The degree of crowdedness in these buses exacerbates with the decline in bus capacity that render passengers willing to choose the earlier/later buses. As bus capacity continues to decrease, more passengers whose desired bus belongs to buses with $\max _{i \in \Lambda}\left\{q_{i}\right\}$ and the neighboring buses consider taking the buses which are dispatched even earlier or later. This pattern of chain reaction will continue in the hypothetical circumstance until bus capacity drops to the true value. It can be verified by inspection that $N_{i}$ in set $\Lambda$ follows the unimodal (not strictly) distribution in the optimal equilibrium solution when $\rho=0$.

Now, let us denote by $\bar{n}=\left\{\bar{n}_{i, j}^{k} \mid i \in \Lambda, j \in \bar{\Lambda}, k=1,2\right\}$ and $\bar{N}=\left\{\bar{N}_{i} \mid i \in \Lambda\right\}$ the equilibrium solution of the lower- 
level model when $\rho$ equals 0 . Meanwhile, we indicate by $\bar{\lambda}_{j}^{k}$ the generalized travel cost of passengers in category $k$ whose desired bus is bus $j \in \bar{\Lambda}$ when $\rho=0$. It is consistent with the special case (see Remark 3 ) that the equilibrium solution is unique with respect to $\bar{\lambda}_{j}^{k}$ and $\bar{N}_{i}$, but not unique with respect to $\bar{n}_{i, j}^{k}$.

In fact, bus with the maximum value of $\bar{N}_{i}\left(\max _{i \in \Lambda}\left\{\bar{N}_{i}\right\}\right)$ is not necessarily bus 0 . Figure 1 depicts four special situations with regard to different distributions of parameter $q_{i}$ for buses in set $\Lambda$. For the ease of observation, we provisionally assume that two delay penalty parameters are equal, i.e., $\beta=\gamma$. As can be seen intuitively, the most crowded bus of the situation in Figure 1(a) at equilibrium is bus 1 , while bus with $\max _{i \in \Lambda}\left\{N_{i}\right\}$ in Figure 1(b) at equilibrium is bus -1 . Due to the full symmetry, there exist two buses with $\max _{i \in \Lambda}\left\{N_{i}\right\}$ in the equilibrium solution for the latter two situations (bus 0 and 1 in Figure 1(c), and bus 0 and -1 in Figure 1(d)).

In this section, we further assume that bus with $\max _{i \in \Lambda}\left\{\bar{N}_{i}\right\}$ is in the set of buses with $\max _{i \in \Lambda}\left\{q_{i}\right\}$. Hereafter, we refer to situations that resemble the former two situations in Figure 1 as Situation 1 and situations similar to the latter two situations in Figure 1 as Situation 2. There is a disparity between two situation types.

In Situation 1, we indicate by $\bar{j}$ the sole bus with $\max _{i \in \Lambda}\left\{N_{i}\right\}$ boarding passengers at equilibrium. Passengers whose desired bus is $\bar{j}$ may shift to either the early arrival buses (e.g., bus $\bar{j}-1$ ) or the late ones (e.g., bus $\bar{j}+1$ ). Yet, for passengers whose desired bus is $\bar{j}-1$, they only consider taking bus $\bar{j}-1$ or earlier buses while passengers whose desired bus is $\bar{j}+1$ only select bus $\bar{j}+1$ or later arrival buses. This shifting behavior is also applicable to bus $i \leq \bar{j}-2$ and bus $i \geq \bar{j}+2$, and the associated chain reaction continues until the in-vehicle crowding effect vanishes.

In Situation 2 , we denote by $\bar{j}_{1}$ and $\bar{j}_{2}$ two most crowded buses at equilibrium, where $\bar{j}_{2}=\bar{j}_{1}+1$. By inspection of Figure $1\left(\mathrm{c}\right.$ ), passengers whose desired bus is $\bar{j}_{1}$ (bus 0 ) do not choose to take bus $\bar{j}_{2}$ (bus 1) or later arrival buses. Similarly, for passengers whose desired bus is $\bar{j}_{2}$, they are not likely to board bus $\bar{j}_{1}$ or earlier buses. At equilibrium, passenger flow forms two isolated branches: passengers whose desired bus $i \leq \bar{j}_{1}$ only select their desired bus or earlier buses, while passengers whose desired bus $i \geq \bar{j}_{2}$ prefer to the desired bus or later buses. In each of two branches, such chain reaction of shifting behavior continues until the buses dispatched earlier or later get no more crowded.

Note that Figure 1 only presents four special situations where we assume $\beta=\gamma$ and demand distribution is ideally symmetric. In practice, the perceived penalty of late arrival is larger than (or equal to) that of early arrival $(\beta \leq \gamma)$ and distribution of parameter $q_{i}$ is asymmetric. These practical circumstances make the shifting behaviors not intuitively apparent.

Situation 1 and Situation 2 can be identified by the mathematical expression in terms of $\bar{N}_{i}$ and $q_{i}(i \in \Lambda)$, as described in Table 2. If there exists one bus $j \in \bar{\Lambda}$ which satisfies $\sum_{i=-\xi}^{j}\left(\bar{N}_{i}-q_{i}\right)<0$ and $\sum_{i=j}^{\zeta}\left(\bar{N}_{i}-q_{i}\right)<0$, the associated equilibrium solution corresponds to Situation 1 . In contrast, the equilibrium solution belongs to Situation 2 if there are two neighboring buses that simultaneously meet the $2^{\text {nd }}$ and $3^{\text {rd }}$ conditions.
Similar to the special case in Section 2.1, it is assumed that there always exist passengers in both two categories taking their desired bus $j$ at equilibrium when $\rho=0$, namely, $\bar{n}_{j, j}^{k}>0$ $(j \in \bar{\Lambda}, k=\{1,2\})$. Hence, $\bar{\lambda}_{j}^{1}=p_{1}+\alpha \tau+C_{j}$ and $\bar{\lambda}_{j}^{2}=p_{2}+$ $\alpha \tau+C_{j}$ hold. Then, we have $\bar{\lambda}_{j}^{1}-p_{1}=\bar{\lambda}_{j}^{2}-p_{2}, j \in \bar{\Lambda}$.

Regarding $\bar{\lambda}_{j}^{k}$ between two different buses, we take the $2^{\text {nd }}$ and $4^{\text {th }}$ conditions of Situation 2 (see Table 2 ) as an example. When bus gets crowded, the first bus that passengers tend to shift is the nearest neighboring bus. In conditions 2 and 4 of Situation 2, for passenger group $k$, this trait is reflected by $\bar{\lambda}_{j}^{k}=p_{k}+\alpha \tau+C_{j}=p_{k}+\alpha \tau+\beta \bar{h}_{k}+C_{j-1}$. Since $\bar{\lambda}_{j-1}^{k}=p_{k}+\alpha \tau+C_{j-1}$, we obtain $\bar{\lambda}_{j}^{k}=\bar{\lambda}_{j-1}^{k}+\beta h$ when $j$ ranges from $-\bar{\xi}$ to $\bar{j}_{1}$. Hence, $\bar{\lambda}_{\bar{j}_{1}}^{k}=\bar{\lambda}_{\bar{j}_{1}-1}^{k}+$ $\beta h=\bar{\lambda}_{\bar{j}_{1}-2}^{k}+2 \beta h=\ldots=\bar{\lambda}_{-\bar{\xi}}^{k}+\delta_{-\bar{\xi}, \bar{j}_{1}}$. Then, for a bus $j \in\left\{-\bar{\xi},-\bar{\xi}+1, \ldots, \bar{j}_{1}\right\}, \quad \bar{\lambda}_{j}^{k}=p_{k}+\alpha \tau+C_{j} \quad=p_{k}+\alpha \tau$ $+\delta_{-\bar{\xi}-1, j}+C_{-\bar{\xi}}$, which implies that the generalized travel cost of taking desired bus $j$ equals that of taking bus $-\bar{\xi}-1$ due to the effect of in-vehicle crowding. Analogously, the generalized travel cost $\bar{\lambda}_{j}^{k}$ associated with the other conditions in Table 2 can be procured, as summarized in Table 3.

Remark 5. As previously stated, some passengers only select their desired bus or earlier buses while some passengers prefer to the desired bus or later buses at equilibrium in both two situation types. It indicates that some values of $\bar{n}_{i, j}^{k}$ constantly equal 0 . In Situation $1, \bar{n}_{i, j}^{k}=0$ always holds when $j \leq \bar{j}-1, i \geq j+1$ or $j \geq \bar{j}+1, i \leq j-1$. In Situation $2, \bar{n}_{i, j}^{k}=$ 0 when $j \leq \bar{j}_{1}, i \geq j+1$ or $j \geq \bar{j}_{2}, i \leq j-1$.

2.4.2. $\rho$ Exceeds 0 . When $\rho$ exceeds 0 , elderly passengers are penalized in terms of pricing surcharge if taking buses in set $\bar{\Lambda}$. Due to the fare increase, more elderly passengers consider shifting to the buses in set $\Lambda \backslash \bar{\Lambda}$, and passengers in two categories are redistributed at equilibrium. Two situations in Section 2.2 are discussed separately.

(1) Situation 1. In Situation 1, for elderly passengers whose desired bus $j$ is in set $\bar{\Lambda}_{1}=\{-\bar{\xi},-\bar{\xi}+1, \ldots, \bar{j}-1\}$, the generalized travel cost of taking bus $j$ equals that of taking bus $-\bar{\xi}-1$ when $\rho=0$ (see Table 3 ). Similarly, for elderly passengers whose desired bus $j \in \bar{\Lambda}_{2}=\{\bar{j}+1, \quad \bar{j}+2$ $\ldots, \bar{\zeta}-1, \bar{\zeta}\}$, the generalized travel cost of taking bus $j$ equals that of taking bus $\bar{\zeta}+1$ when $\rho=0$. Set $\bar{\Lambda}$ equals $\bar{\Lambda}_{1} \cup \bar{\Lambda}_{2} \cup\{\bar{j}\}$.

Once $\rho$ exceeds 0 , elderly passengers begin to shift from buses in set $\bar{\Lambda}$ to buses in set $\Lambda / \bar{\Lambda}$. We concern on the shifting sequence of elderly passengers who originally take buses in either of two sets $\left(\bar{\Lambda}_{1}\right.$ and $\left.\bar{\Lambda}_{2}\right)$ as $\rho$ increases.

Proposition 5. In Situation 1, as $\rho$ gradually increases, the shifting sequence is that elderly passengers forgo taking bus $j$ prior to bus $j+1\left(j, j+1 \in \bar{\Lambda}_{1}\right)$, and instead they select bus $-\bar{\xi}-1$ or earlier buses in the equilibrium solution.

The proof of Proposition 5 is provided in Appendix E. 


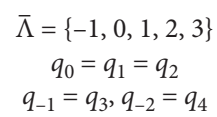

$$
\begin{gathered}
\bar{\Lambda}=\{-3,-2,-1,0,1\} \\
q_{0}=q_{-1}=q_{-2} \\
q_{-3}=q_{1}, q_{-4}=q_{2}
\end{gathered}
$$

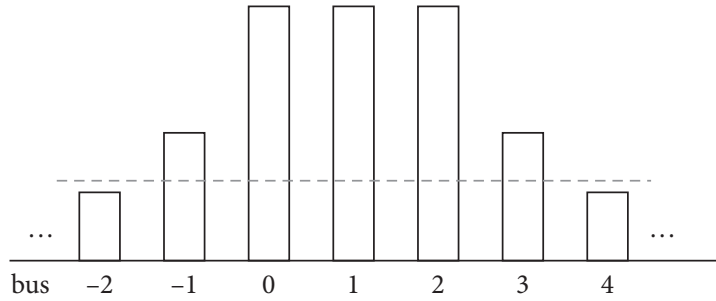

(a)

$\bar{\Lambda}=\{-1,0,1,2$,

$q_{0}=q_{-1}=q_{1}=q_{2}$ $q_{-2}=q_{3}$

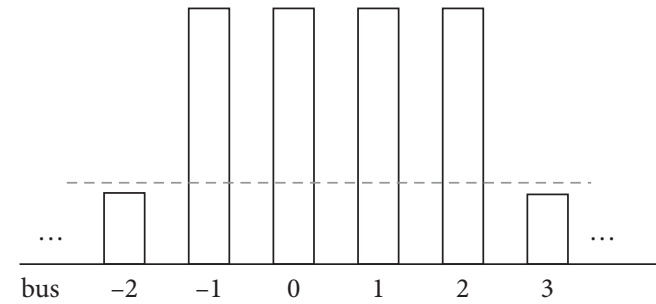

(c)

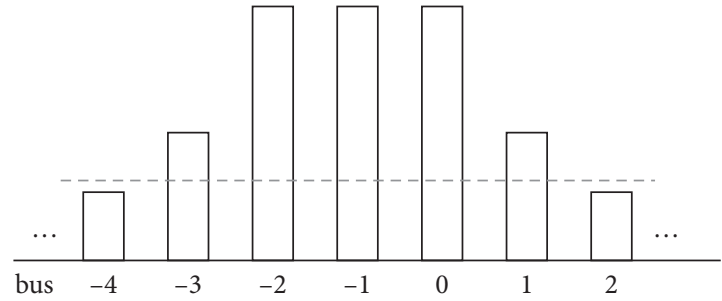

(b)

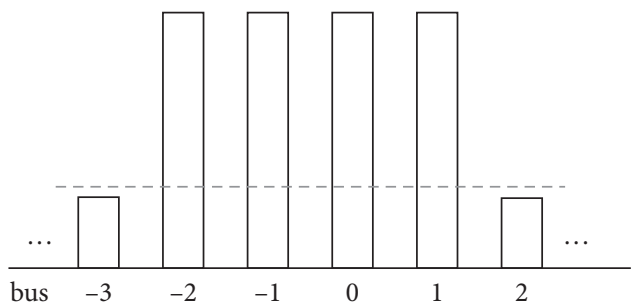

(d)

FIgURE 1: Four special situations with regard to parameter $q_{i}$.

Table 2: Conditions of bus $j(j \in \bar{\Lambda})$ at equilibrium.

\begin{tabular}{lccc}
\hline No. & Condition of bus $j \in \bar{\Lambda}$ & Situation 1 & Situation 1 \\
\hline 1 & $\sum_{i=-\xi}^{j}\left(\bar{N}_{i}-q_{j}\right)<0, \sum_{i=j}^{\xi}\left(\bar{N}_{i}-q_{j}\right)<0$ & $j=\bar{j}$ & - \\
2 & $\sum_{i=\xi}^{j}\left(\bar{N}_{i}-q_{j}\right)<0, \sum_{i=j}^{\xi}\left(\bar{N}_{i}-q_{j}\right)<0$ & - & $j=\bar{j}_{1}$ \\
3 & $\sum_{i=-\xi}^{j}\left(\bar{N}_{i}-q_{j}\right)<0, \sum_{i=j}^{\xi}\left(\bar{N}_{i}-q_{j}\right)=0$ & - & $j=\bar{j}_{2}=\bar{j}_{1}+1$ \\
4 & $\sum_{i=-\xi}^{j}\left(\bar{N}_{i}-q_{j}\right)<0, \sum_{i=j}^{\xi}\left(\bar{N}_{i}-q_{j}\right)<0$ & $j \leq \bar{j}-1$ & $j \leq \bar{j}_{1}-1$ \\
5 & $\sum_{i=-\xi}^{j}\left(\bar{N}_{i}-q_{j}\right)<0, \sum_{i=j}^{\xi}\left(\bar{N}_{i}-q_{j}\right)>0$ & $j \leq \bar{j}+1$ & $j \geq \bar{j}_{1}+1$ \\
\hline
\end{tabular}

TABLE 3: Generalized travel cost of two situation types.

\begin{tabular}{lc}
\hline Situation 1 & $j \in \bar{\Lambda}=\{-\bar{\xi},-\bar{\xi}+1, \ldots, \bar{j}, \ldots, \bar{\zeta}-1, \bar{\zeta}\}$ \\
$j=\bar{j}_{1}$ & $\bar{\lambda}_{j}^{k}=p_{k}+\alpha \tau+C_{j}=p_{k}+\alpha \tau+\delta_{-\bar{\xi}_{-1, j}}+C_{-\bar{\xi}_{-1}}=p_{k}+\alpha \tau+\delta_{\bar{\xi}_{+1, j}}+C_{\bar{\xi}_{+1}}$ \\
$j \leq \bar{j}-1$ & $\bar{\lambda}_{j}^{k}=p_{k}+\alpha \tau+C_{j}=p_{k}+\alpha \tau+\delta_{-\bar{\xi}_{-1, j}}+C_{-\bar{\xi}-1}$ \\
$j \geq \bar{j}+1$ & $\bar{\lambda}_{j}^{k}=p_{k}+\alpha \tau+C_{j}=p_{k}+\alpha \tau+\delta_{\bar{\xi}_{+1, j}}+C_{\bar{\xi}_{+1}}$ \\
Situation 2 & $j \in \bar{\Lambda}=\left\{-\bar{\xi},-\bar{\xi}+1, \ldots, \bar{j}_{1}, \bar{j}_{2}, \ldots, \bar{\zeta}-1, \bar{\zeta}\right\}$ \\
$j \leq \bar{j}_{1}$ & $\bar{\lambda}_{j}^{k}=p_{k}+\alpha \tau+C_{j}=p_{k}+\alpha \tau+\delta_{-\bar{\xi}-1, j}+C_{-\bar{\xi}_{-1}}$ \\
$j \geq \bar{j}_{1}$ & $\bar{\lambda}_{j}^{k}=p_{k}+\alpha \tau+C_{j}=p_{k}+\alpha \tau+\delta_{\bar{\xi}_{+1, j}}+C_{\bar{\xi}_{+1}}$ \\
\hline
\end{tabular}

Lemma 3. In Situation 1, as $\rho$ incrementally increases, the shifting sequence is that elderly passengers forgo taking bus $j$ prior to bus $j-1\left(j, j-1 \in \bar{\Lambda}_{2}\right)$, and instead they select bus $\bar{\zeta}+1$ or later buses in the equilibrium solution.

For elderly passengers whose desired bus is $\bar{j}$, their generalized travel cost of taking bus $\bar{j}$ equals that of taking both bus $-\bar{\xi}-1$ and $\bar{\zeta}+1$ when $\rho=0$. With the increase of $\rho$, the behavior of elderly passengers whose desired bus is $\bar{j}$ may evolve into the following three subsituations:

${ }_{-2}^{2}$ Situations 1.1: $\quad \bar{\lambda}_{\bar{j}}^{2}=p_{2}+\alpha \tau+\delta_{-\bar{\xi}-1, \bar{j}}+C_{-\bar{\xi}_{-1}} \quad$ and $\bar{\lambda}_{\bar{j}}^{2}=p_{2}+\alpha \tau+\delta_{\bar{\zeta}+1, \bar{j}}+C_{\bar{\zeta}+1}$ always hold as $\rho$ increases. It indicates that elderly passengers whose desired bus is $\bar{j}$ are 
willing to shift to either the early departure bus $-\bar{\xi}-1$ or the late departure bus $\bar{\zeta}+1$.

Situations 1.2: $\quad \bar{\lambda}_{\bar{j}}^{2}=p_{2}+\alpha \tau+\delta_{-\bar{\xi}-1, \bar{j}}+C_{-\bar{\xi}-1} \quad$ and $\bar{\lambda}_{\bar{j}}^{2}=p_{2}+\alpha \tau+\delta_{\bar{\zeta}+1, \bar{j}}+C_{\bar{\zeta}+1}$ hold at the outset, whereas $\bar{\lambda}_{\bar{j}}^{2}<p_{2}+\alpha \tau+\delta_{-\bar{\xi}-1, \bar{j}}+C_{-\bar{\xi}-1}$ when $\rho$ is increased to a certain value (denoted by $\widehat{\rho}_{\bar{j}}$ ). Differed from Situations 1.1, when $\rho \geq \widehat{\rho}_{\bar{j}}$, two isolated passenger flow branches exist, which is equivalent to the initial Situation 2 , where bus $\bar{j}-1$ and $\bar{j}$ correspond to bus $\bar{j}_{1}$ and $\bar{j}_{2}$ in Situation 2, respectively.

Situations 1.3: $\quad \bar{\lambda}_{\bar{j}}^{2}=p_{2}+\alpha \tau+\delta_{-\bar{\xi}-1, \bar{j}}+C_{-\bar{\xi}-1} \quad$ and $\bar{\lambda}_{\bar{j}}^{2}=p_{2}+\alpha \tau+\delta_{\bar{\zeta}_{+1, \bar{j}}}+C_{\bar{\zeta}_{+1}}$ hold at the outset, whereas $\bar{\lambda}_{\bar{j}}^{2}<p_{2}+\alpha \tau+\delta_{\bar{\zeta}_{+1, \bar{j}}}+C_{\bar{\zeta}+1}$ when $\rho$ is increased to a certain value (denoted by $\breve{\rho}_{\bar{j}}$ ). Differed from Situations 1.1, when $\rho \geq \breve{\rho}_{\bar{j}}$, two isolated passenger flow branches exist, which is equivalent to the initial Situation 2 , where bus $\bar{j}$ and $\bar{j}+1$ correspond to bus $\bar{j}_{1}$ and $\bar{j}_{2}$ in Situation 2, respectively.

In either of three above subsituations, it is easy to verify that the most crowded bus at equilibrium is always bus $\bar{j}$ since we assume that bus with $\max _{i \in \Lambda}\left\{\bar{N}_{i}\right\}$ (namely, $\left.N_{\bar{j}}\right)$ is in the set of buses with $\max _{i \in \Lambda}\left\{q_{i}\right\}$. Meanwhile, the value of $N_{\bar{j}}$ monotonically decreases as $\rho$ increases until none of elderly passengers take buses in set $\bar{\Lambda}$ or $\rho$ attains the maximum value (i.e., $p_{1}-p_{2}$ ).

(1) Situation 2. In the initial Situation 2, passenger flow forms two isolated branches between buses $\bar{j}_{1}$ and $\bar{j}_{2}$ in the equilibrium solution when $\rho=0$. Passengers whose desired bus is $\bar{j}_{1}\left(\bar{j}_{2}\right)$ are unwilling to take bus $\bar{j}_{2}\left(\bar{j}_{1}\right)$, which can be expressed mathematically as $C_{\bar{j}_{1}}+\gamma h>C_{\bar{j}_{2}}$ and $C_{\bar{j}_{1}}<C_{\bar{j}_{2}}+\beta h$. With the increase of $\rho$, more elderly passengers proceed to shift to buses in set $\Lambda / \bar{\Lambda}$, and Situation 2 may evolve into the following three subsituations:

Situations 2.1: $C_{\bar{j}_{1}}+\gamma h>C_{\bar{j}_{2}}$ and $C_{\bar{j}_{1}}<C_{\bar{j}_{2}}+\beta h$ always hold as $\rho$ increases. Two isolated passenger flow branches exist throughout. Specifically, passengers whose desired bus $j \leq \bar{j}_{1}$ only choose their desired bus or earlier buses, while passengers whose desired bus $j \geq \bar{j}_{2}$ choose their desired bus or later buses.

Situations 2.2: $C_{\bar{j}_{1}}+\gamma h>C_{\bar{j}_{2}}$ holds as $\rho$ increases, whereas $C_{\bar{j}_{1}}=C_{\bar{j}_{2}}+\beta h$ when $\rho$ attains a certain value (denoted by $\widetilde{\rho}_{\bar{j}_{1}}$ ). In the case of $\rho<\widetilde{\rho}_{\bar{j}_{1}}$, two isolated passenger flow branches exist. When $\rho \geq \widetilde{\rho}_{j_{1}}^{-}$, this subsituation is equivalent to the initial Situation 1 , in which bus $\bar{j}_{1}$ corresponds to bus $\bar{j}$ in Situation 1 .

Situations 2.3: $C_{\bar{j}_{1}}<C_{\bar{j}_{2}}+\beta h$ holds as $\rho$ increases, whereas $C_{\bar{j}_{1}}+\gamma h=C_{\bar{j}_{2}}$ when $\rho$ attains a certain value (denoted by $\widetilde{\rho}_{\bar{j}_{2}}$ ). If $\rho<\widetilde{\rho}_{\bar{j}_{2}}$, two isolated passenger flow branches exist. When $\rho \geq \widetilde{\rho}_{j_{2}}^{-}$, this subsituation is identical to the initial Situation 1 , in which bus $\bar{j}_{2}$ corresponds to bus $\bar{j}$ in Situation 1.

In either of three above subsituations, it can be verified that the most crowded bus at equilibrium is either bus $\bar{j}_{1}$ or bus $\bar{j}_{2}$, and the value of $\max \left(N_{\bar{j}_{1}}, N_{\bar{j}_{2}}\right)$ monotonically decreases as $\rho$ increases until none of elderly passengers select buses in set $\bar{\Lambda}$ or $\rho$ reaches the maximum value (i.e., $p_{1}-p_{2}$ ).

\section{Solution Method}

The proposed bilevel EPS model aims to determine the minimum amount of fare increase $\rho$ for elderly passengers who select buses in set $\bar{\Lambda}$. The rationale is to delivery one value of $\rho$ to the lower-level model [LM]. Then, model [LM] obtains the optimal equilibrium solution in terms of $\mathbf{N}=\left\{N_{i} \mid i \in \Lambda\right\}$, which is the feedback to the upper-level model [UM].

Essentially, elderly pricing surcharge scheme is designed to alleviate the degree of in-vehicle crowdedness. It is reflected in constraint (4) of model [UM] that the number of passengers taking bus $i$ (i.e., $N_{i}$ ) should be not more than $\phi Q$. In fact, we only need to guarantee that the most crowded bus satisfies equation (4).

In this study, we develop a bisection-based trial-and-error algorithm to solve the bilevel model, on the grounds that it is difficult to procure some key parameters such as the crowding cost function $g(\cdot)$ in equation (2). In contrast, given the value of $\rho$, observing the number of passengers taking bus $i \in \Lambda$ (i.e., $N_{i}$ ) at equilibrium seems to be more practicable.

3.1. Special Case. First, the special case with one bus in set $\bar{\Lambda}$ is discussed. We need to identify the price adjustment mechanism based upon each trial and observation accordingly, so as to garner the optimal value of $\rho$ (denoted by $\rho^{*}$ ). The trial-and-error algorithm to identify $\rho^{*}$ is described as follows.

Initially, we let $\rho^{(1)}$ equal $p_{1}-p_{2}$, which is the maximum value in model [UM]. Though the crowding cost function and some other parameters are unknown, the number of invehicle passengers at equilibrium $N_{i}^{(1)}(i \in \Lambda)$ can be observed by field observation after implementing $\rho^{(1)}$.

Then, we check whether the bilevel model is feasible by comparing $N_{0}^{(1)}$ with $\phi Q$ (because bus 0 is always the most crowded bus). If $N_{0}^{(1)}>\phi Q$, the model is infeasible in terms of constraint (4). As stated in Proposition 4, the special case $(\bar{\Lambda}=\{0\})$ is classified into two scenarios, which can be identified by the relation between $N_{0}^{(1)}$ and $\bar{N}_{0}$. If $N_{0}^{(1)}=\bar{N}_{0}$, the case is Scenario I; otherwise, it is Scenario II. Note that Scenario I is constantly infeasible because $\bar{N}_{0}$ exceeds $\phi Q$. Therefore, we only focus on Scenario II and $N_{0}^{(1)} \leq \phi Q$, which ensure that the model has at least one feasible solution.

Regarding Scenario II, $N_{0}$ is monotonically decreasing in $\rho$ according to Proposition 4 . Our task is to find the minimum value of $\rho$ satisfying $\max _{i \in \Lambda}\left\{N_{i}\right\} \leq \phi Q$. Due to the monotonicity, $\rho$ attains the optimal (i.e., $\rho^{*}$ ) when $\max _{i \in \Lambda}\left\{N_{i}\right\}=\phi Q$. In the next iteration, the solution method proceeds to solve model [LM] when $\rho^{(2)}$ equals the midpoint of interval $\left[0, \rho^{(1)}\right]$. Later, model $[\mathrm{LM}]$ returns the optimal equilibrium solution $N_{i}^{(2)}(i \in \Lambda)$ to model [UM]. If $N_{i}^{(2)}>\phi Q$, then $\rho^{*}>\rho^{(2)}$, meaning that the interval $\left[0, \rho^{(2)}\right]$ can be discarded, and the updated interval will be $\left[\rho^{(2)}, \rho^{(1)}\right]$. If $N_{0}^{(1)} \leq \phi Q$, then $\rho^{*} \leq \rho^{(2)}$, and the search can focus on $\left[0, \rho^{(2)}\right]$. The bisection-based trial-and-error mechanism is to conduct iterations following the above procedure until the size of the remaining interval is less than a given accuracy.

The process of the algorithm is presented in Algorithm 1. 
Step 1: set the iteration number $\kappa=0$. Let $\rho^{(\kappa)}=0$ and obtain the result of $\bar{N}_{i}(i \in \Lambda)$. Set $\kappa=\kappa+1$. Step 1: let $\rho^{(\kappa)}$ equal $p_{1}-p_{2}$. Solve model [LM] and obtain the optimal equilibrium solution $N_{i}^{(\kappa)}(i \in \Lambda)$. For bus 0 , if $N_{0}^{(\kappa)}>\phi Q$, the model has no feasible solution; otherwise, let the initial interval $\left[\rho_{\mathrm{LB}}, \rho_{\mathrm{UB}}\right]$ equal $\left[\rho^{(\kappa-1)}, \rho^{(\kappa)}\right]$ and go to Step 2.

Step 2: set $\kappa=\kappa+1$. Let $\rho^{(\kappa)}$ be the midpoint of the current interval. Solve model [LM] and obtain the current optimal equilibrium solution $N_{i}^{(\kappa)}(i \in \Lambda)$.

Step 3: if $N_{0}^{(\kappa)}>\phi Q$, then $\rho^{*}>\rho^{(\kappa)}$, meaning that the interval $\left[\rho^{(\kappa-2)}, \rho^{(\kappa)}\right]$ can be discarded, and the updated interval $\left[\rho_{\mathrm{LB}}, \rho_{\mathrm{UB}}\right]$ will be $\left[\rho^{(\kappa)}, \rho^{(\kappa-1)}\right]$. If $N_{0}^{(\kappa)} \leq \phi Q$, then $\rho^{*} \leq \rho^{(\kappa)}$, and update the interval $\left[\rho_{\mathrm{LB}}, \rho_{\mathrm{UB}}\right]$ as $\left[\rho^{(\kappa-2)}, \rho^{(\kappa)}\right]$.

Step 4: check whether the size of the updated interval is less than a given accuracy (denoted by $\varepsilon$ ). If $\rho_{\mathrm{UB}}-\rho_{\mathrm{LB}} \leq \varepsilon, \rho_{\mathrm{UB}}$ is the approximately optimal solution and stop; otherwise, go to Step 2.

Algorithm 1: Bisection-based trial-and-error algorithm.

3.2. Generic Case. Now, we focus on the solution method of the generic case with multiple buses in set $\bar{\Lambda}$. It is a bit different from the special case in that the generic case exists two various situations, which may further evolve into various subsituations as the value of $\rho$ changes.

In Situation 1 , bus $\bar{j}$ is always the most crowded bus (i.e., $\left.\max _{i \in \Lambda}\left\{N_{i}\right\}\right)$ at equilibrium as $\rho$ increases, and $N_{\bar{j}}$ experiences a monotonic decreases as $\rho$ increases until $\sum_{j \in \bar{\Lambda}} n_{j, \bar{j}}^{2}=0$. Hence, we only need to find the bus with $\max _{i \in \Lambda}\left\{\bar{N}_{i}\right\}$ when $\rho=0$, which corresponds to bus $\bar{j}$.

In Situation 2, there are two most crowded buses when $\rho=0$, namely, bus $\bar{j}_{1}$ and $\bar{j}_{2}$. Regardless of which subsituation it may evolve into, the value of $\max \left(N_{\bar{j}_{1}}, N_{\bar{j}_{2}}\right)$ is monotonically decreasing (not strictly) in $\rho$ until none of elderly passengers select buses in set $\bar{\Lambda}$.

Actually, two situations are not intuitive to be distinguished. At the same time, either situation may further evolve into various subsituations as $\rho$ increases. Nevertheless, we propose one approach that simultaneously fits in two situations. Firstly, it is consistent with Situation 1 that we seek out the most crowded bus when $\rho=0$ (which is still denoted by $\bar{j}$ ). Then, three neighboring buses (bus $\bar{j}-1, \bar{j}$, and $\bar{j}+1$ ) compose one bus subset, denoted by $\bar{\Lambda}_{\bar{j}}$ (when Situation 2 takes place, bus $\bar{j}_{1}$ and $\bar{j}_{2}$ must be in subset $\bar{\Lambda}_{\bar{j}}$, which correspond to either the former two neighboring buses $(\bar{j}-1$ and $\bar{j}$, respectively) or the latter two buses $(\bar{j}$ and $\bar{j}+1$, respectively)). It can be verified that in both two situations, $\max _{j \in \bar{\Lambda}_{-}^{-}}\left(N_{j}\right)$ is monotonically decreasing (or at least nonincreasing) in $\rho$ until elderly passengers no longer select buses in set $\bar{\Lambda}$ or $\rho$ reaches the maximum value (i.e., $p_{1}-p_{2}$ ).

On this basis, the proposed algorithm in Algorithm 1 can be employed to address the generic case through minor adjustments: let $N_{0}^{(\kappa)}$ and $\bar{N}_{0}$ be substituted by $\max _{j \in \bar{\Lambda}_{\bar{i}}}\left(N_{j}^{(\kappa)}\right)$ and $\max _{j \in \bar{\Lambda}_{\bar{j}}}\left(\bar{N}_{j}\right)$, respectively, from Step 1 to Step 3. It is worth noticing that the modified trial-and-error algorithm is suitable for both two situations and potentiallyrealizable subsituations in the generic case.

\section{Numerical Examples}

The proposed model and solution method are verified using two examples which are performed on a personal computer with Intel Core i7-4700 CPU and 8.00 G RAM. Two numerical examples are based upon one express bus service in Yancheng bus terminal, located at the Tinghu District of
Yancheng, China. A total of five-workday morning peak demand data were obtained from field investigations of the ticket hall at bus terminal. Related parameters are set as follows: for the express bus service, suppose that the invehicle travel time $\tau$ is taken as $0.5 \mathrm{~h}$ and bus headway is $0.1 \mathrm{~h}$. The following values of cost parameters are used: the unit cost of travel time $\alpha=10(\$ / \mathrm{h})$, the unit penalty cost parameters $(\beta, \gamma)=(18,20)(\$ / h)$, and the initial ticket prices of two passenger categories are $\left(p_{1}, p_{2}\right)=(6,3)(\$ / \mathrm{h})$.

For buses $i \in \Lambda / \bar{\Lambda}, q_{i}^{1}$ and $q_{i}^{2}$ are set to equal 20 and 10 , respectively. The unknown crowding cost function $g(\cdot)$ is utilized to simulate the choices of passengers in two categories after each trial of pricing surcharge $\rho$, described as follows:

$$
g\left(N_{i}\right)= \begin{cases}0, & N_{i} \leq N_{s}, \\ -\vartheta \times \ln \left(1-\frac{N_{i}-N_{s}}{Q-N_{s}+\zeta}\right), & N_{s}<N_{i} \leq Q,\end{cases}
$$

where $N_{i}$ is the number of in-vehicle passengers taking bus $i \in \Lambda$. Vehicle capacity $Q$ is taken as 90 , and $N_{s}$ denotes the number of seats, which is taken as 30 . Parameters $\vartheta$ and $\zeta$ are set to be 4 and 0.01 , respectively.

4.1. Example 1. We first consider a simple example which corresponds to the special case with one bus in set $\bar{\Lambda}$, i.e., $\bar{\Lambda}=\{0\}$. As previously stated in Section 2, parameter $\phi$ indicates the threshold of bus crowdedness degree, and $\phi Q$ in equation (4) represents the maximum number of invehicle passengers, which is intimately related with the level of service. Figure 2 exhibits the results of elderly pricing surcharge scheme for different values of $q_{0}$ when $\phi$ is taken as 0.7 and 0.8 , respectively. Here, the number of elderly passengers $q_{0}^{2}$ is supposed to be fixed, which equals 40 .

Pricing surcharge scheme does not work on all the values of $q_{0}$. For instance, when $\phi=0.7$, the range of $q_{0}$ can be divided into three areas, as described on the left-hand side of Figure 2(a). In the green shaded area, there is no need to implement the pricing surcharge scheme because the number of passengers at equilibrium for most crowded bus 0 (i.e., $N_{0}$ ) does not exceed $\phi Q$ when $\rho=0$. The blue shaded area represents the effective interval where pricing surcharge scheme is indeed in effect. In such area, $q_{0}$ ranges from 64 to 103 when $\phi=0.7$, and its interval length happens to equal $q_{0}^{2}$. 

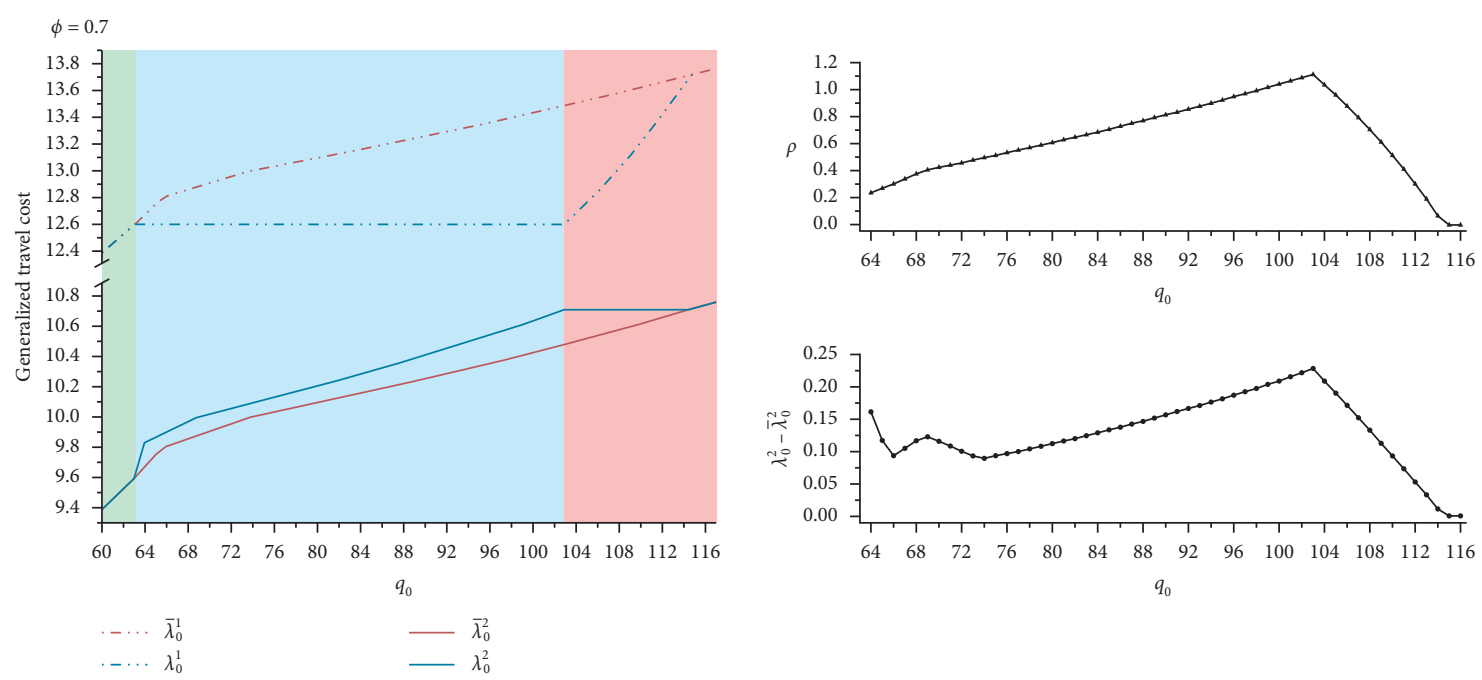

(a)
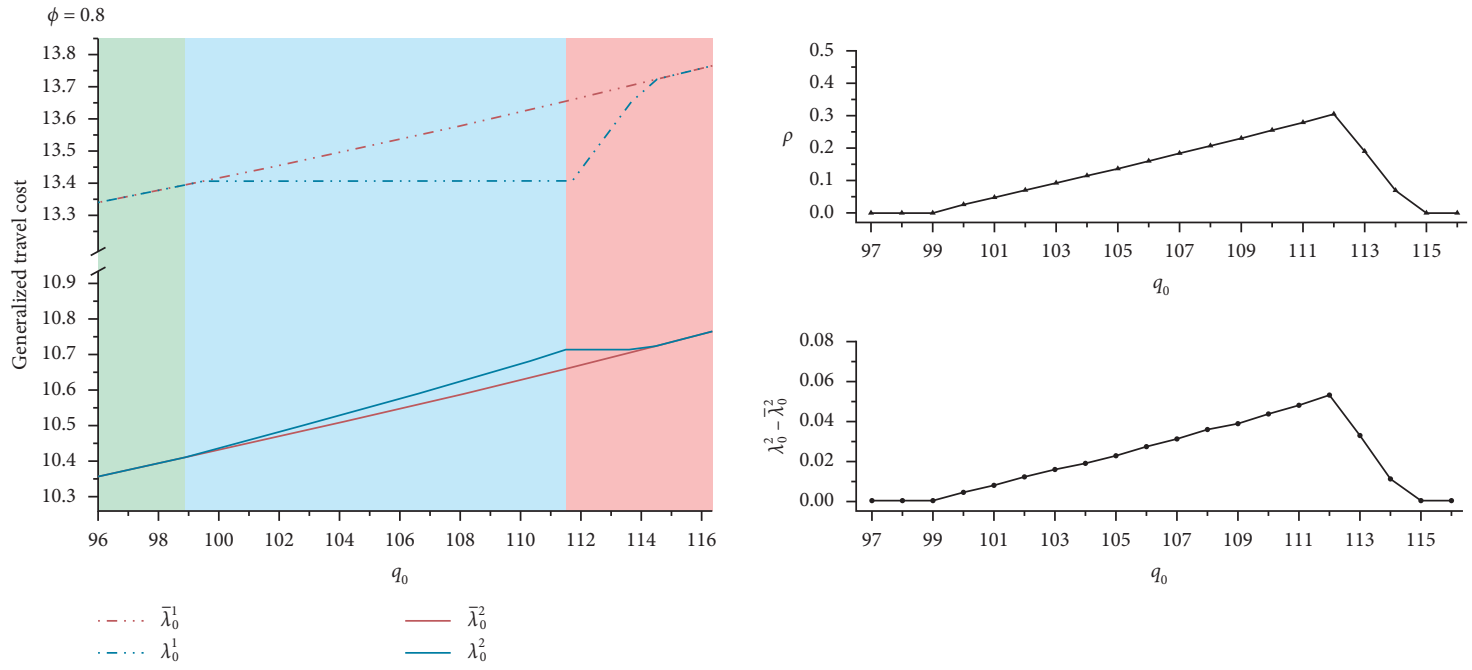

(b)

Figure 2: Results of example 1.

Meanwhile, $\rho$ attains the optimal value when equation (4) is binding, which monotonically increases from 0.24 \$ to 1.12 \$ as $q_{0}$ increases from 64 to 103 . It is worth noting that for the elderly passengers taking bus 0 , the resulting growth of generalized travel cost $\left(\lambda_{0}^{2}-\bar{\lambda}_{0}^{2}\right)$ is significantly lower than $\rho$, as shown on the right-hand side of Figure 2(a).

The red shaded area denotes the interval in which the proposed bilevel model has no feasible solution since equation (4) cannot be satisfied. This area can be further divided into two conditions depending on the relation between $\bar{N}_{0}$ and $q_{0}^{1}$. The first condition is that $q_{0}$ ranges from 104 to 115 . In such condition, $q_{0}^{1} \leq \bar{N}_{0}$ when $\rho=0$ which corresponds to Scenario II as described in Section 2.1. Though pricing surcharge strategy fails to directly meet the required level of service in equation (4), it is still conducive to alleviating the in-vehicle congestion to some extent. Yet, $\bar{N}_{0}$ increases as $q_{0}$ increases. Then, the potentially influenced passengers (elderly passengers who take bus 0 ) by implementing pricing surcharge scheme get less, and the value of $\rho$ sharply drops off to 0 when $q_{0}$ attains 115 . Therefore, the benefit of elderly pricing surcharge fades away with the growth of $q_{0}$. The second condition is $q_{0}>115$ in which $q_{0}^{1}>\bar{N}_{0}$ when $\rho=0$. It corresponds to Scenario I in Section 2.1. According to Remark 4, $\rho$ is irrelevant to the generalized travel cost of two passenger categories, and hence, elderly pricing surcharge scheme becomes useless.

When $\phi=0.8$, the effective interval (the blue shaded area) is apparently narrowed as compared to the case of $\phi=0.7$. It is because when $q_{0}$ ranges from 72 to $98, N_{0}$ in the equilibrium solution in case of $\rho=0$ (i.e., $\bar{N}_{0}$ ) is less than $\phi Q=72$. It indicates that though $q_{0}$ exceeds $\phi Q$, passengers spontaneously select the neighboring buses due to in-vehicle congestion of their desired bus 0 without conducting pricing surcharge scheme. Hence, this interval belongs to the green shaded area. At the same time, the optimal value of $\rho$ when $\phi=0.8$ is generally less than that when $\phi=0.7$. Moreover, 
TABle 4: Demand parameters of buses $i \in \bar{\Lambda}$ in example 2.

\begin{tabular}{|c|c|c|c|c|c|}
\hline \multirow{4}{*}{ Case a } & Bus $i \in \bar{\Lambda}$ & -2 & -1 & 0 & 1 \\
\hline & $q_{i}^{1}$ & 50 & 60 & 80 & 70 \\
\hline & $q_{i}^{2}$ & 20 & 30 & 30 & 30 \\
\hline & $q_{i}$ & 70 & 90 & 110 & 100 \\
\hline \multirow{4}{*}{ Case b } & Bus $i \in \bar{\Lambda}$ & -2 & -1 & 0 & 1 \\
\hline & $q_{i}^{1}$ & 50 & 50 & 80 & 70 \\
\hline & $q_{i}^{2}$ & 20 & 20 & 30 & 30 \\
\hline & $q_{i}$ & 70 & 70 & 110 & 100 \\
\hline \multirow{4}{*}{ Case c } & Bus $i \in \bar{\Lambda}$ & -1 & 0 & 1 & 2 \\
\hline & $q_{i}^{1}$ & 70 & 80 & 50 & 40 \\
\hline & $q_{i}^{2}$ & 30 & 30 & 20 & 20 \\
\hline & $q_{i}$ & 100 & 110 & 70 & 60 \\
\hline \multirow{4}{*}{ Case $\mathrm{d}$} & Bus $i \in \bar{\Lambda}$ & -1 & 0 & 1 & 2 \\
\hline & $q_{i}^{1}$ & 70 & 80 & 60 & 40 \\
\hline & $q_{i}^{2}$ & 30 & 30 & 30 & 20 \\
\hline & $q_{i}$ & 100 & 110 & 90 & 60 \\
\hline
\end{tabular}

the more the pricing surcharge is imposed on elderly passengers, the higher the level of service in terms of in-vehicle crowdedness bus 0 can get at equilibrium within the blue shaded area.

4.2. Example 2. We now pay attention to the generic case with multiple buses in set $\bar{\Lambda}$. In example 2 , set $\bar{\Lambda}$ contains four buses. Table 4 lists four different cases and associated demand parameters of each bus in set $\bar{\Lambda}$. The crowdedness threshold parameter $\phi$ in equation (4) is set to be 0.9 .

Figure 3 presents the results of $N_{i}$ and $C_{i}$ with respect to four different cases in example 2. As can be seen, these four cases embody various situation types either when $\rho$ equals 0 or when $\rho$ attains the optimal value $\rho^{*}$ :

Case a: when $\rho$ equals 0 , bus 0 is the most crowded bus and the shifting behavior of passengers at equilibrium is consistent with Situation 1 described in Section 2.2. For elderly passengers whose desired bus is bus 0 , the generalized travel cost of taking early arrival bus (bus -3 ) and late arrival bus (bus 2) is identical. Once pricing surcharge strategy is performed, elderly passengers increasingly shift to buses in set $\Lambda \backslash \bar{\Lambda}$ as $\rho$ increases. Case a evolves into Situation 1.2 in which $\widehat{\rho}_{\bar{j}}=1.22$ is the demarcation point. The blue line in Figure 3(a) depicts the distribution of $C_{i}$ when $\rho$ equals 1.21. Once $\rho \geq 1.22$, two isolated passenger flow branches arise. Passengers whose desired bus is bus -1 or -2 only consider taking bus -3 or even earlier buses, while passengers whose desired bus is bus 0 or 1 only consider bus 2 or even later arrival buses. The optimal value $\rho^{*}$ equals 2.81, and its equilibrium results of $N_{i}$ and $C_{i}$ correspond to the green part in Figure 3(a). It signifies that elderly passengers need to pay $5.81 \$$ when they wish to take buses $i \in \bar{\Lambda}$ in the morning rush hour.

Case b: similar to Case a, the shifting behavior of passengers at equilibrium is Situation 1 when $\rho=0$. Case b evolves into Situation 1.1, which means elderly passengers whose desired bus is bus 0 prefer to take either bus -3 or bus 2 in set $\Lambda / \bar{\Lambda}$ as $\rho$ increases. When $\rho$ attains 2.70, the bilevel model is optimal since the number of in-vehicle passengers in most crowded bus (i.e., bus 0 ) at equilibrium equals $\phi Q$.

Case c: coherent with Cases a and b, bus 0 is the most crowded bus, and situation type of passenger shifting behavior is Situation 1 when $\rho=0$. Differed from the above two cases, Case c evolves into Situation 1.3 in which $\widehat{\rho}_{j}=1.75$ is the demarcation point. The blue part drawn in Figure 3(c) describes the distribution of $C_{i}$ and $N_{i}$ given that $\rho$ equals 1.74. When $\rho \geq 1.75$, there exist two isolated passenger flow branches. It means that elderly passengers whose desired bus is bus 0 only consider shifting to bus -2 or even earlier buses. In this case, the optimal pricing surcharge $\rho^{*}$ equals 2.27.

Case d: from the outset, passenger flow in Case $d$ forms two isolated branches between bus 0 and bus 1 when $\rho=0$, which corresponds to Situation 2. Afterwards, Case d evolves into Situation 2.1, which means that two isolated passenger flow branches exist throughout as $\rho$ increases. Passengers whose desired bus $i \leq 0$ prefer their desired bus or earlier buses, while passengers whose desired bus $i \geq 1$ prefer to choose their desired bus or later buses. In this case, the bilevel model is optimal when $\rho^{*}$ equals 2.27 .

Additionally, a sensitivity analysis is conducted on the effect of demand parameters. Case a differs from Case $b$ in $q_{-1}^{1}$ and $q_{-1}^{2}$ of bus -1 . Here, $q_{-1}^{2}$ is supposed to be fixed, which equals 30 . Table 5 presents the variation of situation type with respect to changes in $q_{-1}^{1}$. When the values of $q_{-1}^{1}$ are in different interval, the associated situation types are different. Note that two intervals $([45,72]$ and $[73,80])$ share the same $\rho^{*}$. It is because when $\rho=\rho^{*}$, there exist two isolated passenger flow branches. Bus -1 is located in the less crowded branch, whereas $\rho^{*}$ is dependent upon the other branch which are identical for these two intervals. Analogously, the difference between Case $c$ and Case $d$ is the values of $q_{1}^{1}$ and $q_{1}^{2}$ of bus 1 . Here, $q_{1}^{2}$ is supposed to be fixed, which equals 30 . There are also three 


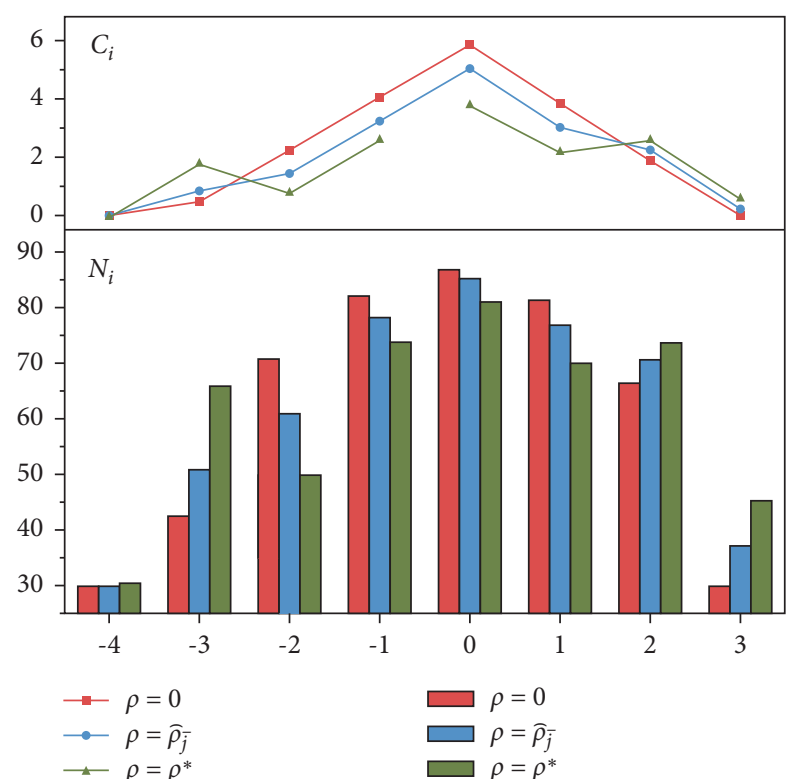

(a)

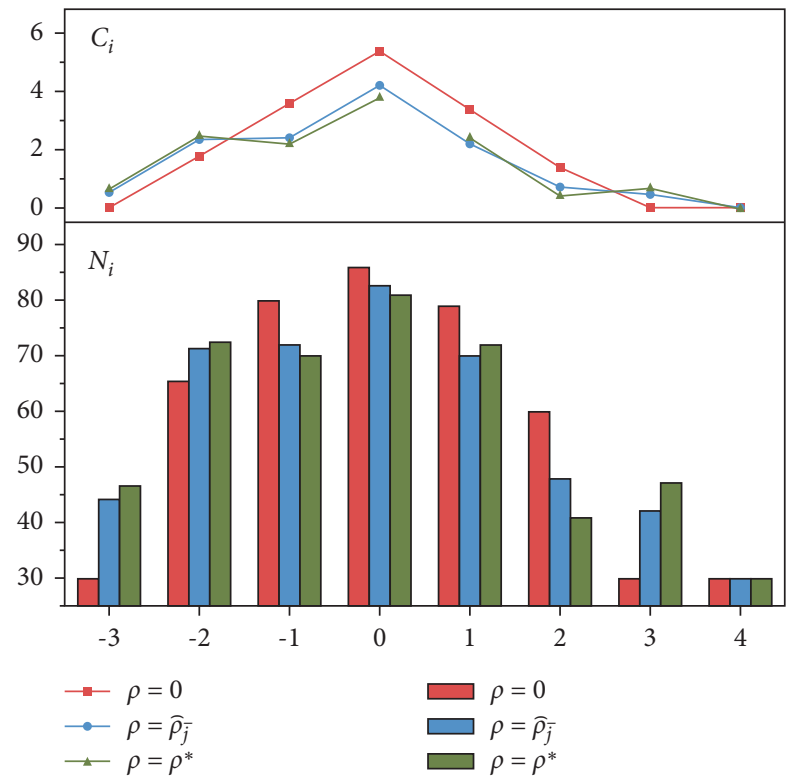

(c)
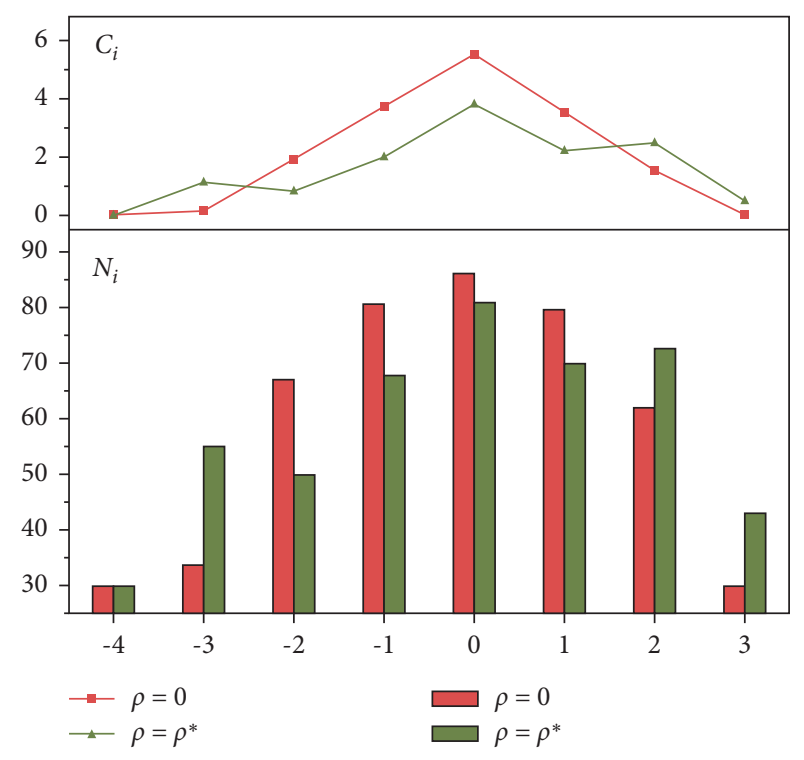

(b)

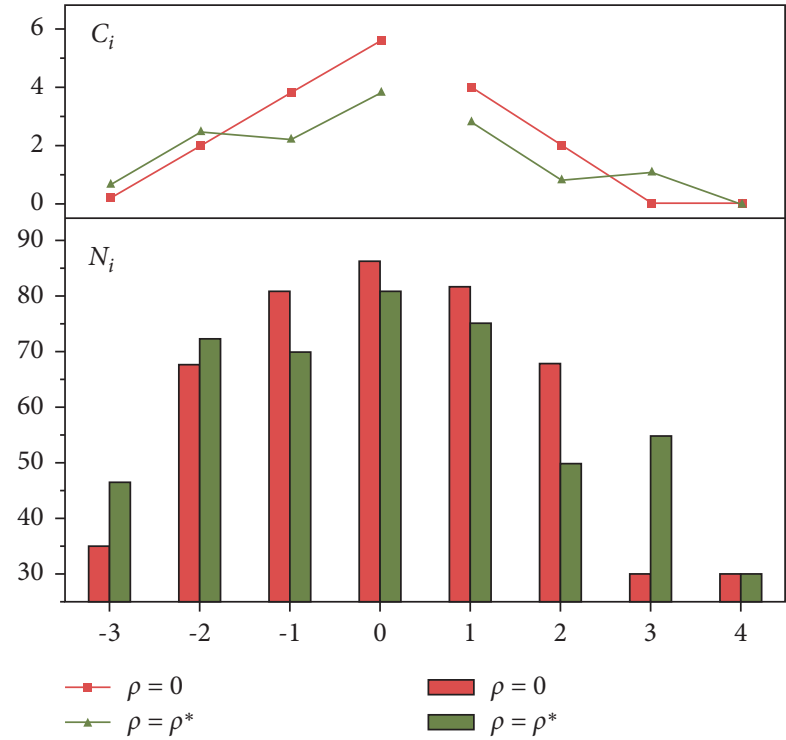

(d)

Figure 3: Results of $N_{i}$ and $C_{i}$ in example 2.

TABLE 5: Situation type with respect to changes in demand parameters.

\begin{tabular}{lcc}
\hline & Case $(\mathrm{a}, \mathrm{b})$ & \\
\hline$q_{i=-1}^{1}$ & Situation type $(\rho=0)$ & Situation type $(\rho>0)$ \\
{$[40,44]$} & Situation 1 & Situation 1.1 \\
{$[45,72]$} & Situation 1 & Situation 1.2 \\
{$[73,80]$} & Situation 2 & Situation 2.1 \\
\hline & Case $(\mathrm{c}, \mathrm{d})$ & Situation type $(\rho>0)$ \\
$q_{i=1}^{1}$ & Situation type $(\rho=0)$ & Situation 1.1 \\
{$[30,35]$} & Situation 1 & Situation 1.3 \\
{$[36,52]$} & Situation 1 & Situation 2.1 \\
{$[53,80]$} & Situation 2 & \\
\hline
\end{tabular}


possible situation types in regard to the changes in $q_{1}^{1}$, as described in Table 5.

\section{Conclusion}

This study focused on the elderly fare pricing issue for taking express buses in the morning peak period. Compared with the current fare discount policy, imposing surcharge on the elderly is a potentially beneficial instrument that encourages partial elderly passengers to circumvent the most crowded buses. The elderly pricing surcharge (EPS) problem was formulated as a bilevel optimization model, in which the upper-level model is to make the pricing surcharge decision, and the lower-level model is the equilibrium passenger assignment that represents passengers' bus choice behavior. The proposed model of EPS was classified into the special case and the generic case depending on the number of buses that impose surcharge. We analyzed several useful properties of two cases and developed a trial-and-error solution method to solve these two cases. Numerical examples present that the elderly pricing surcharge scheme does not fit in all the demand scenarios, which has a certain effective interval. Moreover, we found that for elderly passengers, the resulting increase of generalized travel cost due to surcharge is apparently lower than the surcharge $\rho$ per se. This study could serve as one of the management tools for alleviating the in-vehicle crowding of express buses during the morning peak period.

Admittedly, our proposed model comes with some limitations, and the following improvements are suggested: (i) passengers are assumed to have the identical value of travel time and valuation of in-vehicle crowding, and it could be considered to extend the model by incorporating the discrete choice model to reflect passengers with heterogeneous characteristics; (ii) overcrowding, namely, the case of passengers who fail to board is not considered in this study, and research is needed to propose a more generalized model which is capable to consider overcrowding issue that passengers who do not get on a bus continue to wait for the next bus; (iii) unimodal distribution assumption could be relaxed to consider other different distributions; and (iv) the elastic demand could be incorporated into the model as it is possible to lose passengers or attract new users when user acceptability is considered (i.e., passengers may decide to use a different mode of travel or forgo their trips). The authors recommend that future studies could focus on these issues.

\section{Appendix}

\section{A. When $\boldsymbol{\rho}>0, n_{i=0, j=0}^{1}>0$ always holds in the optimal equilibrium solution}

Proof. It is proven below by contradiction. Consider the case that $n_{i=0, j=0}^{1}=0$ in the optimal equilibrium solution. As $q_{0}^{1}>0$, passengers in the first category shift to the neighboring buses. Assume that some of these passengers choose bus $i \in \Lambda / \bar{\Lambda}$, namely, $n_{i \neq j, j=0}^{1}>0$. Based on equations (14) and (15), $\quad \lambda_{j=0}^{1}=p_{1}+\alpha \tau+\delta_{i \neq j, j=0}+C_{i} \quad$ and $\lambda_{j=0}^{1} \leq p_{1}$ $+\alpha \tau+C_{0}$. Hence, $C_{0} \geq \delta_{i \neq j, j=0}+C_{i}>0$, which means that $N_{i=0}>0$.

For the elderly, $p_{2}+\rho+\alpha \tau+C_{0} \geq p_{2}+\rho+\alpha \tau+\delta_{i \neq j, j=0}+$ $C_{i}>p_{2}+\alpha \tau+\delta_{i \neq j, j=0}+C_{i}$ given that $\rho>0$. It signifies that $n_{i=0, j=0}^{2}=0$. Based on equation (8), $N_{i=0}=n_{i=0, j=0}^{1}+n_{i=0, j=0}^{2}=$ 0 which yields a contradiction because $N_{i=0}>0$.

B. If passengers whose desired bus $j=0$ select bus $i \leq-2$ (i.e., $n_{i, j=0}^{1}+n_{i, j=0}^{2}>0$ ), the equilibrium generalized travel costs of taking bus $i^{\prime}=i, \ldots,-2,-1$ are identical for each passenger category $\boldsymbol{k}(k \in\{1,2\})$

Proof. We prove this proposition by contradiction. Suppose that there exist some passengers whose desired bus $j=0$ choose bus $i(i \leq-2)$, whereas the generalized travel cost of taking bus $i$ and $i+1$ is not the same. Apparently, the associated cost of bus $i$ is lower than bus $i+1$; otherwise, passengers whose desired bus $j=0$ would not select bus $i$.

First, consider the case that $n_{i, j=0}^{1}>0(k=1)$. Based on equations (14) and (15), we obtain that $\lambda_{j=0}^{1}=p_{1}+\alpha \tau+(-i) \beta h+C_{i}<p_{1}+\alpha \tau+(-i-1) \beta h+C_{i+1}$, and $n_{i+1, j=0}^{1}=0$. Then, $C_{i}+\beta h<C_{i+1}$. For the elderly, $p_{2}+$ $\alpha \tau+(-i) \beta h+C_{i}<p_{2}+\alpha \tau+(-i-1) \beta h+C_{i+1}$ which means $n_{i+1, j=0}^{2}=0$. Based on equation (8), $N_{i+1}=q_{i+1}$, and hence, bus $i+1$ is an uncrowded bus.

Passengers prefer to take their desired bus when it is in an uncrowded condition, even though the neighboring buses are empty. This character can be expressed mathematically as $\tau g\left(\max _{i \in i \in \Lambda / \bar{\Lambda}} q_{i}\right)<\min (\beta h, \gamma h)$, because $\max _{i \in i \in \Lambda / \Lambda} q_{i}$ passengers cannot make one bus be crowded if no other passengers select it. Since $N_{i+1}=q_{i+1}, C_{i+1}=\tau g$ $\left(q_{i+1}\right)<\min (\beta h, \gamma h) \leq \beta h$ which yields a contradiction as $C_{i}+\beta h<C_{i+1}$.

The case of $n_{i, j=0}^{2}>0(k=2)$ can be proved in an analogous way. The above procedure is implemented recursively to obtain that the generalized travel cost of taking bus $i^{\prime}=$ $i, \ldots,-2,-1$ is identical.

\section{For passengers whose desired bus is in set $i \in \Lambda \bar{\Lambda}$, the assumption that they are always available to board their desired bus can be relaxed, with no influence on the objective value of the lower-level model}

Proof. When passengers in set $\Lambda$ do not shift to bus $i$ in set $i \in \Lambda / \bar{\Lambda}$ (i.e., $n_{i, j=0}^{1}+n_{i, j=0}^{2}=0$ ), it is intuitive that all the $q_{i}$ passengers can take their desired bus. In case that $n_{i, j=0}^{1}+$ $n_{i, j=0}^{2}>0$ and $i \leq-2$, we obtain that $C_{i+1}=\beta h+C_{i}$ according to Proposition 2. Let $m_{i} \leq \min \left(n_{i, j=0}^{1}+n_{i, j=0}^{2}, q_{i+1}\right)$. Suppose that $m_{i}$ passengers whose desired bus is bus 0 while selecting bus $i$ at equilibrium and $m_{i}$ passengers whose desired bus is bus $i+1$ exchange positions with each other. This operation yields no change in terms of $N_{i}$ and $N_{i+1}$ in equation (8), and hence, the in-vehicle crowding cost term in equation (6) is also fixed. Meanwhile, due to $C_{i+1}=\beta h+C_{i}$, it is easy to 
verify that the generalized travel cost of passengers whose desired bus is either 0 or $i+1$ keeps the same as the original result at equilibrium. Similarly, passengers whose desired bus $i^{\prime}=i+1, \ldots,-2,-1$ can take the earlier bus. It fits the situation in $n_{i, j=0}^{1}+n_{i, j=0}^{2}>0$ and $i \geq 2$ as well. Even though partial passengers whose desired bus $i^{\prime}=1,2, \ldots, i-1$ select the later bus, the objective function value of the lower-level model can be invariant at equilibrium.

D. Scenario I $\left(q_{0}^{1}-\bar{n}_{i=0, j=0}^{1}>\bar{n}_{i=0, j=0}^{2}\right)$, $\lambda_{j=0}^{1}-p_{1}=\lambda_{j=0}^{2}-p_{2}$ and $n_{i=0, j=0}^{2}=0$ hold at equilibrium given that $\rho>0$; Scenario II $\left(q_{0}^{1}-\bar{n}_{i=0, j=0}^{1} \leq \bar{n}_{i=0, j=0}^{2}\right), \lambda_{j=0}^{1}-p_{1} \geq \lambda_{j=0}^{2}-p_{2}-\rho$ and $\sum_{i \in \Lambda / \Lambda} n_{i, j=0}^{1}=0$ hold at equilibrium given that $\rho>0$

Proof. According to the relation between $q_{0}^{1}-\bar{n}_{i=0, j=0}^{1}$ and $\bar{n}_{i=0, j=0}^{2}$, we distinguish the following two scenarios.

Scenario I: $q_{0}^{1}-\bar{n}_{i=0, j=0}^{1}>\bar{n}_{i=0, j=0}^{2}$.

We provide a proof of this scenario by contradiction. Assume that $n_{i=0, j=0}^{2}>0$ at equilibrium when $\rho>0$. According to Proposition 1, $n_{i=0, j=0}^{1}>0$. At equilibrium, $\lambda_{j=0}^{1}=p_{1}+\alpha \tau+C_{0}$ and $\lambda_{j=0}^{2}=p_{2}+\rho+\alpha \tau+C_{0}$. By these two equations, we obtain $p_{2}=\lambda_{j=0}^{2}-\lambda_{j=0}^{1}+p_{1}-\rho$.

For an arbitrary bus $i \in \Lambda / \bar{\Lambda}$, equation (19) presents that $\lambda_{j=0}^{2} \leq p_{2}+\alpha \tau+\delta_{i, j}+C_{i}$. Substituting $p_{2}, \quad \lambda_{j=0}^{2} \leq\left(\lambda_{j=0}^{2} \quad-\right.$ $\left.\lambda_{j=0}^{1}+p_{1}-\rho\right)+\alpha \tau+\delta_{i, j}+C_{i}$, and then $\lambda_{j=0}^{1} \leq p_{1}+$ $\alpha \tau+\delta_{i, j}+C_{i}-\rho$. Due to $\rho>0, \lambda_{j=0}^{1}<p_{1}+\alpha \tau+\delta_{i, j}+C_{i}$. We obtain that $n_{i, j=0}^{1}=0$ based on equation (14). It is easy to verify that $n_{i=0, j=0}^{1}=q_{i=0}^{1}$ at equilibrium. $C_{i}(\rho>0)=$ $\tau g\left(N_{i}\right)=\tau g\left(q_{0}^{1}+n_{i=0, j=0}^{2}\right)>\tau g\left(q_{0}^{1}\right)$. In contrast, $C_{i}(\rho=0)$ $=\tau g\left(\bar{n}_{i=0, j=0}^{1}+\bar{n}_{i=0, j=0}^{2}\right)<\tau g\left(q_{0}^{1}\right)$. Therefore, the minimal generalized travel cost of passenger category $k$ at equilibrium (denoted by $\lambda_{j=0}^{k}(\rho>0)$ ) is larger than $\bar{\lambda}_{j=0}^{k}$.

Next, based on $\bar{n}_{i, j=0}^{k}$ and $\bar{\lambda}_{j=0}^{k}$ when $\rho=0$, we devise a feasible solution for the case of $\rho>0$. As $\bar{n}_{i=0, j=0}^{2} \leq q_{0}^{1}-\bar{n}_{i=0, j=0}^{1}=\sum_{i \in \Lambda / \Lambda} n_{i, j=0}^{1}$, let $\bar{n}_{i=0, j=0}^{2}$ elderly passengers who take bus 0 exchange positions with $\bar{n}_{i=0, j=0}^{2}$ passenger with full-price ticket whose desired bus is bus 0 while selecting bus $i \in \Lambda / \bar{\Lambda}$. This operation enables that the objective function value of model [L2] does not change, and meantime, the modified solution becomes one feasible equilibrium solution for the case of $\rho>0$. We denote by $\hat{\lambda}_{j=0}^{\mathrm{s}}(\rho>0)$ the generalized travel cost of passengers with full-price ticket whose desired bus is bus 0 , which equals $\bar{\lambda}_{j=0}^{1}$.

We get $\widehat{\lambda}_{j=0}^{1}(\rho>0)<\lambda_{j=0}^{1}(\rho>0)$. This contradicts with the fact that $\lambda_{j=0}^{k=0}(\rho>0)$ is the minimal generalized travel cost of passenger category $k$ at equilibrium. Thus, $n_{i=0, j=0}^{2}=0$ holds given that $\rho>0$. At the same time, we can easily verify that the above modified solution is the optimal equilibrium solution for the case of $\rho>0$, and $\lambda_{j=0}^{1}-p_{1}=\lambda_{j=0}^{2}-p_{2}$ holds.

Scenario II: $q_{0}^{1}-\bar{n}_{i=0, j=0}^{1} \leq \bar{n}_{i=0, j=0}^{2}$.

We also consider a proof by contradiction. First, one feasible solution is designed for the case of $\rho \longrightarrow 0^{+}$. As $q_{0}^{1}-$ $\bar{n}_{i=0, j=0}^{1} \leq \bar{n}_{i=0, j=0}^{2}$ and $q_{0}^{1}=\sum_{i \in \Lambda} \bar{n}_{i, j=0}^{1}, \quad \sum_{i \in \Lambda / \Lambda} \bar{n}_{i, j=0}^{1} \leq \bar{n}_{i=0, j=0}^{2}$.
Based on $\bar{n}_{i, j=0}^{k}$ and $\bar{\lambda}_{j=0}^{k}$, let all the $\sum_{i \in \Lambda / \Lambda} \bar{n}_{i, j=0}^{1}$ passengers with full-price ticket whose desired bus is bus 0 while taking other buses in set $\Lambda / \bar{\Lambda}$ exchange positions with $\sum_{i \in \Lambda / \Lambda} \bar{n}_{i, j=0}^{1}$ elderly passengers who take bus 0 . By this operation, the objective function value of model [L2] and the generalized travel cost of two passenger categories do not change when $\rho=0$. In the modified solution, $\widetilde{n}_{i=0, j=0}^{1}=q_{0}^{1}, \widetilde{n}_{i=0, j=0}^{2}=$ $\bar{n}_{i=0, j=0}^{2}-\sum_{i \in \Lambda / \Lambda} \bar{n}_{i, j=0}^{1}$, and $\tilde{\lambda}_{j=0}^{k}=\bar{\lambda}_{j=0}^{k}$.

Now suppose there exists an arbitrary bus $i \in \Lambda / \bar{\Lambda}$ that $n_{i, j=0}^{1}>0$. According to Proposition 1, $n_{i=0, j=0}^{1}>0$. At equilibrium, $\lambda_{j=0}^{1}=p_{1}+\alpha \tau+C_{0}=p_{1}+\alpha \tau+\delta_{i, j}+C_{i}$, it means $C_{0}=\delta_{i, j}+C_{i}$. Then, $p_{2}+\rho+\alpha \tau+C_{0}>p_{2}+\alpha \tau+\delta_{i, j}+C_{i}$ as $\rho>0$. Based on equation (16), $n_{i=0, j=0}^{2}=0$ and $\sum_{i \in \Lambda / \Lambda} n_{i, j=0}^{2}=q_{0}^{2}$. Compared with the above modified solution $\left(\sum_{i \in \Lambda / \Lambda} n_{i, j=0}^{2}=q_{0}^{2}-n_{i=0, j=0}^{2}\right)$, it is intuitive that the generalized travel cost of passengers with full-price ticket whose desired bus is bus 0 while taking bus $i$ is larger than $\widetilde{\lambda}_{j=0}^{1}$ since more elderly passengers shift to buses in set $\Lambda / \bar{\Lambda}$. Moreover, passengers taking bus 0 equals $n_{i=0, j=0}^{1}$ which is less than $\widetilde{n}_{i=0, j=0}^{1}+\widetilde{n}_{i=0, j=0}^{2}$, and hence, $\lambda_{j=0}^{1}<\tilde{\lambda}_{j=0}^{1}$. Obviously, bus 0 is a better option for associated $n_{i, j=0}^{1}$ passengers with full-price ticket, which yields the contradiction. Thus, $\sum_{i \in \Lambda / \Lambda} n_{i, j=0}^{1}=0$ when $\rho>0$.

Based upon Proposition 1 and equation (14), $\lambda_{j=0}^{1}=p_{1}+$ $\alpha \tau+C_{0}$. Equation (17) indicates that $\lambda_{j=0}^{2} \leq p_{1}+\rho+\alpha \tau+C_{0}$. Hence, in Scenario II, $\lambda_{j=0}^{1}-p_{1} \geq \lambda_{j=0}^{2}-p_{2}-\rho$ hold at equilibrium given that $\rho>0$.

\section{E. In Situation 1, as $\rho$ gradually increases, the shifting sequence is that elderly passengers forgo taking bus $j$ prior to bus $j+1$ $\left(j, j+1 \in \bar{\Lambda}_{1}\right)$, and instead they select bus $-\bar{\xi}-1$ or earlier buses in the equilibrium solution}

Proof. It is proven by contradiction. Suppose that there exists one value of $\rho$ (denoted by $\tilde{\rho}$ ) that some elderly passengers select bus $j\left(j \in \bar{\Lambda}_{1} /\{\bar{j}-1\}\right)$, whereas none of elderly passengers are willing to take bus $j+1$. Mathematically, we have $\sum_{j^{\prime}=j}^{\bar{j}} n_{j, j^{\prime}}^{2}>0$ and $\sum_{j^{\prime}=j+1}^{\bar{j}} n_{j+1, j^{\prime}}^{2}=0$.

Based on Remark 1 , the optimal solution is not unique with respect to $\mathbf{n}$. Since $\sum_{j^{\prime}=j}^{\bar{j}} n_{j, j^{\prime}}^{2}>0$, it is easy to verify that one optimal solution $\mathbf{n}$ exists which satisfies $n_{j, j}^{2}>0$. With equation (16) and Table $3, \bar{\lambda}_{j}^{2}=p_{2}+\alpha \tau+\delta_{-\bar{\xi}_{-1, j}}+C_{-\bar{\xi}_{-1}}=$ $p_{2}+\widetilde{\rho}+\alpha \tau+C_{j}$ holds. Meanwhile, elderly passengers shift from bus $j+1$ to bus $-\bar{\xi}-1$ or earlier buses because of the higher generalized travel cost. Namely, $n_{j+1, j+1}^{2}=0$ and $\bar{\lambda}_{j+1}^{2}=p_{2}+\alpha \tau+\delta_{-\bar{\xi}-1, j+1}+C_{-\bar{\xi}-1}<p_{2}+\tilde{\rho}+\alpha \tau+C_{j+1}$. Then, we obtain $C_{j}+\beta h<C_{j+1}$.

For passengers whose desired bus is bus $j^{\prime} \in\{j+1$, $j+2, \ldots, \bar{j}\}, p_{1}+\alpha \tau+\delta_{j+1, j^{\prime}}+C_{j+1}>, p_{1}+\alpha \tau+\delta_{j, j^{\prime}}+C_{j}$, and $p_{2}+\widetilde{\rho}+\alpha \tau+\delta_{j+1, j^{\prime}}+C_{j+1}>\quad p_{2}+\widetilde{\rho}+\alpha \tau+\delta_{j, j^{\prime}}+C_{j}$ given that $C_{j}+\beta h<C_{j+1}$. It means that $n_{j+1, j^{\prime}}^{1}=0$ and $n_{j+1, j^{\prime}}^{2}=0$ hold. Hence, $N_{j+1}=\sum_{k=1}^{2} \sum_{j^{\prime}=j+1}^{\bar{j}} n_{j+1, j^{\prime}}^{k}=0$ which yields a contradiction because $N_{j+1}>0$. 
As can be seen, elderly passengers firstly forgo taking bus $-\bar{\xi}$ and then bus $-\bar{\xi}+1, \ldots, \bar{j}-1$ in sequence in set $\bar{\Lambda}_{1}$ with the continuous increase of $\rho$.

\section{Conflicts of Interest}

The authors declare that they have no conflicts of interest.

\section{Acknowledgments}

This study was supported by the National Natural Science Foundation of China (no. 71901059), the Natural Science Foundation of Jiangsu Province in China (BK20180402), and the Fundamental Research Funds for the Central Universities (2242021R10126 and 2242021R10068).

\section{References}

[1] Q. An, X. Fu, D. Huang, Q. Cheng, and Z. Liu, "Analysis of adding-runs strategy for peak-hour regular bus services," Transportation Research Part E: Logistics and Transportation Review, vol. 143, Article ID 102100, 2020.

[2] S. Baker and P. White, "Impacts of free concessionary travel: case study of an English rural region," Transport Policy, vol. 17, no. 1, pp. 20-26, 2010.

[3] J. Chen, Z. Liu, S. Zhu, and W. Wang, "Design of limited-stop bus service with capacity constraint and stochastic travel time," Transportation Research Part E: Logistics and Transportation Review, vol. 83, pp. 1-15, 2015.

[4] D. Huang, Y. Gu, S. Wang, Z. Liu, and W. Zhang, "A twophase optimization model for the demand-responsive customized bus network design," Transportation Research Part C: Emerging Technologies, vol. 111, pp. 1-21, 2020.

[5] D. Lei, X. Chen, L. Cheng, L. Zhang, S. V. Ukkusuri, and F. Witlox, "Inferring temporal motifs for travel pattern analysis using large scale smart card data," Transportation Research Part C: Emerging Technologies, vol. 120, Article ID 102810, 2020

[6] Y. Liu, C. Lyu, X. Liu, and Z. Liu, "Automatic feature engineering for bus passenger flow prediction based on modular convolutional neural network," IEEE Transactions on Intelligent Transportation Systems, vol. 22, no. 4, pp. 2349-2358, 2020.

[7] Z. Li and D. Hensher, "Crowding in public transport: a review of objective and subjective measures," Journal of Public Transportation, vol. 16, no. 2, pp. 107-134, 2013.

[8] Q. Tian, H.-J. Huang, and H. Yang, "Equilibrium properties of the morning peak-period commuting in a many-to-one mass transit system," Transportation Research Part B: Methodological, vol. 41, no. 6, pp. 616-631, 2007.

[9] A. Tirachini, D. A. Hensher, and J. M. Rose, "Crowding in public transport systems: effects on users, operation and implications for the estimation of demand," Transportation Research Part A: Policy and Practice, vol. 53, pp. 36-52, 2013.

[10] Y. Zuo, X. Fu, Z. Liu, and D. Huang, "Short-term forecasts on individual accessibility in bus system based on neural network model," Journal of Transport Geography, vol. 93, Article ID 103075, 2021.

[11] R. Alsnih and D. A. Hensher, "The mobility and accessibility expectations of seniors in an aging population," Transportation Research Part a: Policy and Practice, vol. 37, no. 10, pp. 903-916, 2003.
[12] M. O. Olawole and O. Aloba, "Mobility characteristics of the elderly and their associated level of satisfaction with transport services in Osogbo, Southwestern Nigeria," Transport Policy, vol. 35, pp. 105-116, 2014.

[13] W. Y. Szeto, L. Yang, R. C. P. Wong, Y. C. Li, and S. C. Wong, "Spatio-temporal travel characteristics of the elderly in an ageing society," Travel Behaviour and Society, vol. 9, pp. 1020, 2017.

[14] F. Shao, Y. Sui, X. Yu, and R. Sun, "Spatio-temporal travel patterns of elderly people-a comparative study based on buses usage in Qingdao, China," Journal of Transport Geography, vol. 76, pp. 178-190, 2019.

[15] J. Zhou, M. Zhang, and P. Zhu, "The equity and spatial implications of transit fare," Transportation Research Part A: Policy and Practice, vol. 121, pp. 309-324, 2019.

[16] C. Nuworsoo, A. Golub, and E. Deakin, "Analyzing equity impacts of transit fare changes: case study of Alameda-Contra Costa Transit, California," Evaluation and Program Planning, vol. 32, no. 4, pp. 360-368, 2009.

[17] B.-h. Nahmias-Biran, N. Sharaby, and Y. Shiftan, "Equity aspects in transportation projects: case study of transit fare change in Haifa," International Journal of Sustainable Transportation, vol. 8, no. 1, pp. 69-83, 2014.

[18] Z. Ma, A. R. Masoud, and A. O. Idris, "Modeling the impact of transit fare change on passengers' accessibility," Transportation Research Record: Journal of the Transportation Research Board, vol. 2652, no. 1, pp. 78-86, 2017.

[19] H. Yang and Y. Tang, "Managing rail transit peak-hour congestion with a fare-reward scheme," Transportation Research Part B: Methodological, vol. 110, pp. 122-136, 2018.

[20] N. Sharaby and Y. Shiftan, "The impact of fare integration on travel behavior and transit ridership," Transport Policy, vol. 21, pp. 63-70, 2012.

[21] Z.-j. Wang, X.-h. Li, and F. Chen, "Impact evaluation of a mass transit fare change on demand and revenue utilizing smart card data," Transportation Research Part A: Policy and Practice, vol. 77, pp. 213-224, 2015.

[22] Z.-j. Wang, F. Chen, B. Wang, and J.-l. Huang, "Passengers' response to transit fare change: an ex post appraisal using smart card data," Transportation, vol. 45, no. 5, pp. 1559-1578, 2018.

[23] J. Zhang, X. Yan, M. An, and L. Sun, "The impact of beijing subway's new fare policy on riders' attitude, travel pattern and demand," Sustainability, vol. 9, no. 5, p. 689, 2017.

[24] M. Lovrić, S. Raveau, M. Adnan et al., "Evaluating off-peak pricing strategies in public transportation with an activitybased approach," Transportation Research Record, vol. 2544, no. 1, pp. 10-19, 2016.

[25] H.-J. Huang, Q. Tian, and Z.-Y. Gao, “An equilibrium model in urban transit riding and fare polices," in Proceedings of the International Conference on Algorithmic Applications in Management, pp. 112-121, Berlin, Germany, June 2005.

[26] Q. Tian, P. Liu, G. P. Ong, and H. J. Huang, "Morning commuting pattern and crowding pricing in a many-to-one public transit system with heterogeneous users," Transportation Research Part E: Logistics and Transportation Review, vol. 145, Article ID 102182, 2020.

[27] Z. Yang and M. Tang, "Does the increase of public transit fares deteriorate air quality in Beijing?," Transportation Research Part D: Transport and Environment, vol. 63, pp. 49-57, 2018.

[28] W. Chen, L. Cheng, X. Chen, J. Chen, and M. Cao, "Measuring accessibility to health care services for older bus passengers: a finer spatial resolution," Journal of Transport Geography, vol. 93, Article ID 103068, 2021. 
[29] R. C. P. Wong, W. Y. Szeto, L. Yang, Y. C. Li, and S. C. Wong, "Public transport policy measures for improving elderly mobility," Transport Policy, vol. 63, pp. 73-79, 2018.

[30] F. Gaglione, C. Cottrill, and C. Gargiulo, "Urban services, pedestrian networks and behaviors to measure elderly accessibility," Transportation Research Part D: Transport and Environment, vol. 90, Article ID 102687, 2021.

[31] D. Kim, "The transportation safety of elderly pedestrians: modeling contributing factors to elderly pedestrian collisions," Accident Analysis \& Prevention, vol. 131, pp. 268-274, 2019.

[32] A. Ben Jemaa, G. Irato, A. Zanela, A. Brescia, M. Turki, and M. Jaïdane, "Congruent auditory display and confusion in sound localization: case of elderly drivers," Transportation Research Part F: Traffic Psychology and Behaviour, vol. 59, pp. 524-534, 2018.

[33] J. Yang, K. Higuchi, R. Ando, and Y. Nishihori, "Examining the environmental, vehicle, and driver factors associated with crossing crashes of elderly drivers using association rules mining," Journal of Advanced Transportation, vol. 2020, Article ID 2593410, 8 pages, 2020.

[34] S. Lord, M.-S. Cloutier, B. Garnier, and Z. Christoforou, "Crossing road intersections in old age-With or without risks? Perceptions of risk and crossing behaviours among the elderly," Transportation Research Part F: Traffic Psychology and Behaviour, vol. 55, pp. 282-296, 2018.

[35] C. Wei, X. Yan, C. Shao, J. Sun, and L. Ma, "Choice behavior of the elderly regarding street-crossing facility," Journal of Advanced Transportation, vol. 2018, Article ID 3296285, 8 pages, 2018.

[36] G. Newmark, "Reduced fare programs for older adults and persons with disabilities: a peer review of policies," Journal of Public Transportation, vol. 17, no. 2, pp. 165-185, 2014.

[37] R. C. P. Wong, W. Y. Szeto, L. Yang, Y. C. Li, and S. C. Wong, "Elderly users' level of satisfaction with public transport services in a high-density and transit-oriented city," Journal of Transport \& Health, vol. 7, pp. 209-217, 2017.

[38] Y. Yuan, M. Yang, J. Wu, S. Rasouli, and D. Lei, “Assessing bus transit service from the perspective of elderly passengers in Harbin, China," International Journal of Sustainable Transportation, vol. 13, no. 10, pp. 761-776, 2019.

[39] A. Drabicki, O. Cats, R. Kucharski, A. Fonzone, and A. Szarata, "Modelling passengers' willingness to wait in the presence of real-time crowding information (rtci)," Transportmetrica A: Transport Science, vol. 17, 2020.

[40] G. Björklund and J. E. Swärdh, "Estimating policy values for in-vehicle comfort and crowding reduction in local public transport," Transportation Research Part A: Policy and Practice, vol. 106, pp. 453-472, 2017.

[41] M. Beckmann, C. B. Mcguire, and C. B. Winsten, Studies in the Economics of Transportation, Yale University Press, New Haven, CY, USA, 1956.

[42] Y. Sheffi, Urban Transportation Networks, Prentice-Hall, Englewood Cliffs, NY, USA, 1985. 